\title{
Empirical spectral model of wall pressure fluctuations including adverse pressure gradient effects
}

\author{
$\mathrm{Nan} \mathrm{Hu}^{*}$ \\ German Aerospace Center (DLR), Lilienthalplatz 7, D-38108 Braunschweig, Germany
}

\begin{abstract}
An empirical model of the wall pressure fluctuation spectra beneath zero and adverse pressure gradient boundary layers is presented. It is developed based on five separate experimental investigations on four different test facilities, covering a large range of Reynolds number, $2.6 \cdot 10^{3}<R e_{\theta}<1.9 \cdot 10^{4}$. The adverse pressure gradient flows from those experiments were realized on different plate model setups and airfoils. A review of the existing spectral models for adverse pressure gradient boundary layers is given and predictions of those models are compared to the experimental results. Hu and Herr's model is used as the basis for developing the present model. One of the major differences between $\mathrm{Hu}$ and Herr's model and the others is the use of the boundary layer shape factor to evaluate the spectral change at mid-frequencies instead of Clauser's equilibrium parameter. An improvement of the predicted spectral peak location and the respective peak level is made using the present model and its broadband predictions compare well with the experimental results.
\end{abstract}

\section{Nomenclature}

$C_{f} \quad$ skin friction coefficient, $\tau_{w} / Q$

$d p / d x \quad$ pressure gradient

$H \quad$ boundary layer shape parameter, $\delta / \delta^{*}$

$Q \quad$ dynamic pressure, $0.5 \rho U_{0}^{2}$

$R e_{\tau} \quad$ wall shear stress based Reynolds number, $u_{\tau} \delta / \nu$

$R_{\theta} \quad$ boundary layer momentum thickness based Reynolds number, $U_{0} \theta / \nu$

$R_{T} \quad$ time scale ratio, $\delta / U_{e} \cdot \nu / u_{\tau}^{2}$

$R e_{\delta}, R e_{\Delta} \quad$ Reynolds number related to other boundary layer parameters, $(\delta, \Delta) U_{e} / \nu$

$U_{e}, U_{0} \quad$ boundary layer edge velocity and local free-streem velocity

$u_{\tau} \quad$ friction velocity, $\sqrt{\tau_{w} / \rho}$

$\delta \quad$ boundary layer thickness

$\delta^{*} \quad$ boundary layer displacement thickness

$\theta \quad$ boundary layer momentum thickness

$\Delta \quad$ boundary layer defect thickness, $\delta^{*} \sqrt{2 / C_{f}}$

$\Delta_{\delta / \delta^{*}} \quad$ boundary layer related parameter, $\delta / \delta^{*}$

$\beta_{\delta^{*}}, \beta_{\theta} \quad$ boundary layer thickness and displacement thickness based Clauser's equilibreium parameter, $\left(\delta^{*}, \theta\right) / \tau_{w} \cdot d p / d x$

$\beta_{\delta}, \beta_{\Delta} \quad$ Clauser's equilibrium parameter related to other boundary layer parameters, $(\delta, \Delta) / Q \cdot d p / d x$

$\Pi_{\delta^{*}} \quad$ Cole's wake parameter, $0.8 \cdot\left(\beta_{\delta^{*}}+0.5\right)^{3 / 4}$

$\Pi_{\theta} \quad$ Cole's wake parameter related to the boundary layer momentum thickness, $0.8 \cdot\left(\beta_{\theta}+0.5\right)^{3 / 4}$

$\nu \quad$ kinematic viscosity

$\rho \quad$ density

$\tau_{w} \quad$ wall shear stress

$\omega \quad$ angular frequency

*Research scientist, Department of Technical Acoustics, Institute of Aerodynamics and Flow Technology, German Aerospace Center (DLR), nan.hu@dlr.de 


\section{Introduction}

The wall pressure fluctuation field is an important external source for cabin noise and also relevant for some other aeroacoustic problems, such as trailing edge noise. Features of the wall pressure fluctuations have been extensively investigated in theoretical, experimental and numerical studies, ${ }^{1-16}$ however, mostly on zero pressure gradient (ZPG) flows. A comprehensive overview on this subject was provided in the monograph of Blake. ${ }^{17}$ Prediction of the wall pressure spectra is of great practical interest. Many spectral models ${ }^{7,18-22}$ for ZPG boundary layers were proposed. The most used one is Goody's model, ${ }^{22}$ which utilizes self-similarity of wall pressure fluctuation spectra induced by ZPG boundary layers and incorporates Reynolds number effects in the high frequency range with a time scale ratio $R_{T}$, expressed as

$$
\frac{\Phi(\omega) U_{e}}{\tau_{w}^{2} \delta}=\frac{a \cdot\left(\omega \delta / U_{e}\right)^{b}}{\left[\left(\omega \delta / U_{e}\right)^{c}+d\right]^{e}+\left[f R_{T}^{g} \cdot\left(\omega \delta / U_{e}\right)\right]^{h}},
$$

where the value of variables $a-h$ was obtained by fitting measurement data from literature, $a=3, b=2$, $c=0.75, d=0.5, e=3.7, f=1.1, g=-0.57$ and $h=7$. They control the shape of the non-dimensional spectra. Amplitude of the spectra is adjusted by the value of $a$. Slopes in different frequency ranges are driven by a combination of $b, c, e$ and $h$. Variable $b$ fixes the slope at low frequencies, function $c \cdot e-b$ is in charge of the slope at medium frequencies and $h-b$ at high frequencies. The variables $f, g$ combined with $R_{T}$ determine the extension of the mid-frequency range, e.g. a larger $R_{T}$ corresponds to a longer extension of the slope at medium frequencies into higher frequencies. The spectral peak location is affected by the value of $d$. Goody's model spectrum has a shape with a slope of $\omega^{2}$ at low frequencies, $\omega^{-0.775}$ at mid-frequencies and $\omega^{-5}$ at high frequencies.

The spectra induced by non-ZPG boundary layers become more complicated. Experimental stud$\operatorname{ies}^{23-31}$ for pressure gradient effects on wall pressure fluctuations showed that the wall pressure spectra lose their self-similarity. Groups of sensors were installed in streamwise separation distances to measure the spectral development due to the impact of pressure gradients. ${ }^{28,30,31}$ For adverse pressure gradient (APG) boundary layers, the spectral slope at medium frequencies becomes successively steeper moving downsteam. This is because the boundary layer experiences the APG for a longer distance at downstream positions. Furthermore, the stronger the pressure gradient, the steeper the mid-frequency slope is.

Rozenberg et al. ${ }^{32}$ (RRM) analyzed the spectral variation between ZPG and APG from some experimental and numerical results and summarized the changing trends through a combination of boundary layer characteristic variables. Based on the basic form of Goody's model, an empirical spectral model including APG effects was proposed by Rozenberg et al., ${ }^{32}$ expressed as

$$
\frac{\Phi(\omega) U_{e}}{\tau_{w}^{2} \delta^{*}}=\frac{\left[2.82 \Delta_{\delta / \delta_{*}}{ }^{2} \cdot\left(6.13 \Delta_{\delta / \delta_{*}}{ }^{-0.75}+F_{1}\right)^{A_{1}}\right]\left[4.2 \cdot\left(\Pi_{\theta} / \Delta_{\delta / \delta_{*}}\right)+1\right]\left(\omega \delta^{*} / U_{e}\right)^{2}}{\left[4.76 \cdot\left(\omega \delta^{*} / U_{e}\right)^{0.75}+F_{1}\right]^{A_{1}}+\left[8.8 R_{T}^{-0.57} \cdot\left(\omega \delta^{*} / U_{e}\right)\right]^{A_{2}}},
$$

where

$$
\begin{aligned}
F_{1} & =4.76 \cdot\left(1.4 / \Delta_{\delta / \delta_{*}}\right)^{0.75} \cdot\left[0.375 \cdot A_{1}-1\right] \\
A_{1} & =3.7+1.5 \beta_{\theta} \\
A_{2} & =\min \left(3,19 / \sqrt{R_{T}}\right)+7 \\
\beta_{\theta} & =\theta / \tau_{w} \cdot d p / d x \\
\Pi_{\theta} & =0.8 \cdot\left(\beta_{\theta}+0.5\right)^{3 / 4} \\
\Delta_{\delta / \delta_{*}} & =\delta / \delta^{*}
\end{aligned}
$$

Clauser's equilibrium parameter ${ }^{33} \beta_{\theta}$ is used to manage slope variation at medium frequencies, the larger the value of $\beta_{\theta}$, the steeper the slope. The spectra shift to a higher frequency and a larger amplitude as $\Delta_{\delta / \delta_{*}}$ increases. Both $\beta_{\theta}$ and $\Delta_{\delta / \delta_{*}}$ are in charge of the spectral amplitude.

Kamruzzaman et al. ${ }^{34}$ (KBLWK) proposed a spectral model for prediction of the airfoil trailing edge noise. The trailing edge wall pressure fluctuation spectra measured in the vicinity of trailing edge from different investigations ${ }^{27,35-39}$ were used to develop the model, the formulation of the model reads

$$
\frac{\Phi(\omega) U_{e}}{\tau_{w}^{2} \delta^{*}}=\frac{0.45\left[1.75 \cdot\left(\Pi_{\delta^{*}}^{2} \cdot \beta_{\delta^{*}}\right)^{m}+15\right]\left(\omega \delta^{*} / U_{e}\right)^{2}}{\left[\left(\omega \delta^{*} / U_{e}\right)^{1.637}+0.27\right]^{2.47}+\left[\left(1.15 R_{T}\right)^{-2 / 7} \cdot\left(\omega \delta^{*} / U_{e}\right)\right]^{7}},
$$

where $\beta_{\delta^{*}}=\delta_{*} / \tau_{w} \cdot d p / d x, \Pi_{\delta^{*}}=0.8 \cdot\left(\beta_{\delta^{*}}+0.5\right)^{3 / 4}$ and $m=0.5 \cdot(H / 1.31)^{0.3}$. Except for the mid-frequency extension determined by $R_{T}$, the formulated spectrum has a constant shape for different 
pressure gradient configurations, i.e. a constant slope of about $\omega^{-2}$ at medium frequencies and a constant spectral peak location at the non-dimensional frequency. The spectral amplitude is adjusted by a combination of Clauser's equilibrium parameter ${ }^{40} \beta_{\delta^{*}}$, Cole's wake parameter ${ }^{41,42} \Pi_{\delta^{*}}$ and boundary layer shape factor $H$.

Catlett et $a l .^{28,43}$ (CFAS) measured the wall pressure fluctuations on tapered trailing edge sections of a flat plate with three different opening angles and proposed an empirical spectral model based on the measured data, which reads

$$
\begin{aligned}
\frac{\Phi(\omega) U_{e}}{\tau_{w}^{2} \delta}= & \frac{a \cdot\left(\omega \delta / U_{e}\right)^{b}}{\left[\left(\omega \delta / U_{e}\right)^{c}+d\right]^{e}+\left[f R_{T}^{g} \cdot\left(\omega \delta / U_{e}\right)\right]^{h}}, \\
\ln \left(a-a_{G}\right) & =4.98 \cdot\left(\beta_{\Delta} R e_{\Delta}{ }^{0.35}\right)^{0.131}-10.7 \\
b & =2, \\
c-c_{G} & =20.9 \cdot\left(\beta_{\delta} R e_{\delta}^{0.05}\right)^{2.76}+0.162, \\
d-d_{G} & =0.328 \cdot\left(\beta_{\Delta} R e_{\Delta}{ }^{0.35}\right)^{0.310}-0.103, \\
e-e_{G} & =-1.93 \cdot\left(\beta_{\delta} R e_{\delta}{ }^{0.05}\right)^{0.628}+0.172, \\
f-f_{G} & =-2.57 \cdot\left(\beta_{\delta} \operatorname{Re}_{\delta}{ }^{0.05}\right)^{0.224}+1.09 \\
g-g_{G} & =38.1 \cdot\left(\beta_{\delta} H^{-0.5}\right)^{2.11}+0.0276 \\
h-h_{G} & =0.797 \cdot\left(\beta_{\Delta} R e_{\Delta}{ }^{0.35}\right)^{0.0724}-0.310
\end{aligned}
$$

where $\beta_{\delta, \Delta}=(\delta, \Delta) / Q \cdot d p / d x, R e_{\delta, \Delta}=(\delta, \Delta) U_{e} / \nu$ and $\Delta=\delta^{*} \sqrt{2 / C_{f}}$. Parameters $a-h$ are derived by fitting to the measured spectra. The constants $a_{G}-h_{G}$ from Goody's model with correspondent positions in Eq. (4) are replaced with functions of boundary layer parameters except for $b=2$, which stands for an $\omega^{2}$ increase at low frequencies. The spectral amplitude, peak location and slope at medium frequencies are affected by the Clauser's equilibrium parameter and the Reynolds number defined with different length scales.

$\mathrm{Hu} \& \operatorname{Herr}^{31}(\mathrm{HH})$ measured the unsteady pressure on a flat plate where pressure gradients were produced by a rotatable NACA 0012 airfoil installed above the plate. Based on the measured spectra, an empirical spectral model was proposed as

$$
\frac{\Phi(\omega) u_{\tau}}{Q^{2} \theta}=\frac{(81.004 d+2.154) \cdot 10^{-7} \cdot\left(\omega \theta / U_{0}\right)}{\left[\left(\omega \theta / U_{0}\right)^{1.5 h^{1.6}}+d\right]^{1.13 / h^{0.6}}+\left[7.645 R e_{\tau}^{-0.411} \cdot\left(\omega \theta / U_{0}\right)\right]^{6}},
$$

where $\log _{10}(d)=-5.8 \cdot 10^{-5} \cdot R e_{\theta} H-0.35$ and $h=1.169 \ln (H)+0.642$. Three major changes were made in this model compared to the other models. Firstly, instead of $U_{e} / \tau_{w}^{2} \delta\left(\delta^{*}\right), u_{\tau} / Q^{2} \theta$ was used based on a good collapse of the spectral peaks for the measured spectra at ZPG \& APG when scaling with this parameter, whereas over $15 \mathrm{~dB}$ difference between the spectra was found when scaled using $U_{e} / \tau_{w}^{2} \delta$. Hu \& Herr also mentioned a good collapse gained by using parameters $u_{\tau} / Q^{2} \delta$ and $U_{0} / Q^{2} \delta^{*}$, and argued dynamic pressure $Q$ could be more appropriate to scale APG spectra than the usual $\tau_{w}^{2}$ due to the increasing importance of the boundary layer outer layer for the spectral peak. Secondly, the boundary layer shape factor was used to evaluate the spectral slope at medium frequencies instead of Clauser's equilibrium parameter. Overall, the wall pressure fluctuations are mostly affected by the boundary layer mean flow profile and the Reynolds stresses. And the Reynolds stresses are again tightly related to the boundary layer profile. Therefore, the boundary layer profile could be an essential criterion to determine the shape of the wall pressure spectra. The connection between the boundary layer profile and the wall pressure fluctuation spectral shape was demonstrated. On the one hand, the spectrum is almost only affected by the local boundary layer; on the other hand, the local boundary layer parameters are predominantly determined by its upstream history. The measured data illustrated that the shape factor indicates correct trends of the boundary layer profile development for different configurations and streamwise positions whereas the Clauser parameter can fail at different streamwise positions where the pressure gradients change rapidly. An excellent match of the spectral slope at medium frequencies between predictions and measured data was shown using the shape factor as the control parameter. Thirdly, instead of an $\omega^{2}$ increase at low frequencies, a slope of $\omega$ was used in the model which was derived as an averaged value from the measured data and results from literature for the APG cases. Arguments for replacement of the classic $\omega^{2}$ are: 1 , the slope of $\omega^{2}$ is obtained by assuming a frozen flow and only counting the mean-shear source term. However, when dealing with a non-frozen flow the slope becomes flatter because the energy from higher frequencies spreads into lower frequencies. ${ }^{7,44} 2$, wall pressure fluctuation spectra of the turbulence-turbulence source term show a plateau at lower frequencies 
and take over the importance of the mean-shear term in the spectra for a ZPG boundary layer. ${ }^{7,9,11,45-48}$ This can also cause a flatter slope at low frequencies. However, an exact knowledge of the importance of the turbulence-turbulence term for non-ZPG boundary layers is still lacking.

In the present work, five separate experimental investigations of APG boundary layers on four different test facilities are summarized and the experimental results are compared to predictions of the existing models. The APG conditions in the experiments were realized on flat plates with airfoils installed above or with tapered trailing edges and on airfoils, covering a large range of Reynolds number, $2.6 \cdot 10^{3}<$ $R e_{\theta}<1.9 \cdot 10^{4}$. Based on the experimental data and using the basic form of HH's model, a new model is proposed which improves predictions of the spectral peak location and the broadband spectra. A new scaling is used which shows a good collapse of the spectral peak between different experimental datasets.

\section{Experimental data and comparison with models}

Five different experiments to measure wall pressure fluctuations beneath APG boundary layers are selected to investigate the spectral models. Additionally, one beneath ZPG boundary layers is also included. APG boundary layers of the selected experiments were realized on three different conceptions: on a flat plate with airfoils on top of it $\left(\mathrm{Hu} \& \mathrm{Herr}^{31}\right.$ and Schloemer $\left.{ }^{23}\right)$, on tapered trailing edges of a flat plate (Catlett et $a l .{ }^{28,43}$ ) and on airfoils (Suryadi \& $\operatorname{Herr}^{30}$ and Herrig et al. ${ }^{27}$ ). A brief summary of the experimental setups will be provided here. For detailed description of the experiments the reader is referred to the respective papers.

$\mathrm{Hu} \&$ Herr measured the wall pressure fluctuation spectra with pinhole Kulite sensors on a flat plate in the open jet Aeroacoustic Wind Tunnel Braunschweig (AWB). The APG was generated with a rotatable NACA 0012 airfoil installed on top of the plate. Three geometrical angles of attack (AOA) at $6^{\circ}, 10^{\circ}$ and $14^{\circ}$ of the airfoil for realization of the APG were used. Flow parameters were obtained by hot-wire anemometers. Wall pressure spectra beneath ZPG \& APG boundary layers were measured.

Schloemer conducted measurements in the low-turbulence subsonic wind tunnel at Stevens Institute of Technology. A flat plate was installed in the closed test section. Wall pressure spectra were measured with approximately $1.5 \mathrm{~mm}$ diameter flush-mounted microphones on the plate. An APG was achieved by a half NACA 0015 airfoil attached on the top channel wall. Wall pressure spectra and flow properties measured by hot-wire anemometers were only provided for one single position.

Catlett et al. carried out measurements in the open jet section of the Anechoic Flow Facility (AFF) at the Naval Surface Warfare Center, Carderock Division (NSWCCD). Wall fluctuating pressures were measured with instrumented inserts containing flush mounted surface microphones on three different open angle $\left(7^{\circ}, 12^{\circ}\right.$ and $17^{\circ}$ relating to the plate plane) tapered trailing edge sections of a flat plate. Flow parameters were measured by hot-wire anemometers at several different streamwise positions. However, the flow measurements were limited to the wake region only and the mean flow velocity of the inner layer was estimated by a best fit to the theoretical boundary layer profiles.

Suryadi \& Herr measured the wall pressure fluctuations with pinhole Kulite sensors on a DU96 airfoil at chord positions between $x / c=0.77-0.96$ in the AWB. Boundary layer parameters at the measurement positions are evaluated by XFOIL calculations. The values of pressure gradients were derived from the measured data. Data from three streamwise positions on the suction side of the airfoil at two AOAs at $-0.8^{\circ}$ and $3.2^{\circ}$ are collected for the comparison.

Herrig et al. measured the wall fluctuating pressure spectra with flush-mounted $1.6 \mathrm{~mm}$ diameter Kulite sensors with the so called B-screen (eight $0.2 \mathrm{~mm}$ diameter holes around a $1.2 \mathrm{~mm}$ diameter circle) at chord position $x / c \approx 0.99$ on a NACA 0012 airfoil in a closed test section in the Laminar Wind Tunnel (LWT) of the University of Stuttgart. Flow properties are provided by XFOIL calculations. Data from AOAs at $0^{\circ}$ and $4^{\circ}$ on suction side of the NACA 0012 airfoil are collected in this section.

Mean flow properties of turbulent boundary layers from the selected experimental database are summarized in Table 1. In literature, the local free-stream velocity $U_{0}$ is provided for the flat plate boundary layers (the cases of $\mathrm{Hu} \&$ Herr and Schloemer), and the boundary layer edge velocity $U_{e}$ is provided for the boundary layers measured on tapered trailing edges or on airfoils (the cases of Catlett et al., Suryadi \& Herr and Herrig et al.). For convenience, the boundary layer edge velocity is converted into the local free-stream velocity using the relationship $U_{e}=0.99 U_{0}$. The positions listed in the test case of $\mathrm{Hu} \&$ Herr are the distance downstream of leading edge, in the test case of Catlett et al. are the distance upstream of the trailing edge. Boundary layer parameters from the test case of Catlett et al. are acquired by digitizing the plots of measured mean flow properties. ${ }^{43}$ However, pressure gradient values from this test case are not available and these are estimated by making a best-fit to the provided prediction of the CFAS model.

Figs. 1-5 show comparison of the predicted spectra for APG test cases between the models, Fig. 6 for 
Table 1: Boundary layer parameters from the experimental test cases.

\begin{tabular}{|c|c|c|c|c|c|c|c|c|c|c|}
\hline Position & $\begin{array}{c}U_{0} \\
(\mathrm{~m} / \mathrm{s}) \\
\end{array}$ & $\begin{array}{c}\delta \\
(\mathrm{mm})\end{array}$ & $\begin{array}{c}\delta^{*} \\
(\mathrm{~mm})\end{array}$ & $\begin{array}{c}\theta \\
(\mathrm{mm})\end{array}$ & $H$ & $\begin{array}{c}u_{\tau} \\
(\mathrm{m} / \mathrm{s})\end{array}$ & $R e_{\tau}$ & $R e_{\theta}$ & $\begin{array}{c}d p / d x \\
(\mathrm{~Pa} / \mathrm{m})\end{array}$ & $\beta_{\delta^{*}}$ \\
\hline \multicolumn{11}{|c|}{$\mathrm{Hu} \&$ Herr, $\mathrm{AOA}=6^{\circ}$} \\
\hline$x=1128 \mathrm{~mm}$ & 32.3 & 20.0 & 3.88 & 2.56 & 1.52 & 1.03 & 1338 & 5362 & 1156 & 3.5 \\
\hline$x=1210 \mathrm{~mm}$ & 30.8 & 24.4 & 5.61 & 3.49 & 1.61 & 0.89 & 1410 & 6979 & 643 & 3.8 \\
\hline \multicolumn{11}{|l|}{$\mathrm{AOA}=10^{\circ}$} \\
\hline$x=1128 \mathrm{~mm}$ & 32.0 & 23.0 & 5.09 & 3.12 & 1.63 & 0.88 & 1314 & 6492 & 1225 & 6.8 \\
\hline$x=1210 \mathrm{~mm}$ & 30.4 & 28.7 & 7.68 & 4.39 & 1.75 & 0.745 & 1388 & 8670 & 518 & 6.0 \\
\hline \multicolumn{11}{|l|}{$\mathrm{AOA}=14^{\circ}$} \\
\hline$x=1128 \mathrm{~mm}$ & 31.2 & 26.3 & 7.38 & 3.87 & 1.91 & 0.645 & 1102 & 7831 & 1084 & 16.2 \\
\hline$x=1210 \mathrm{~mm}$ & 29.9 & 35.0 & 12.07 & 5.69 & 2.12 & 0.51 & 1159 & 11046 & 320 & 12.5 \\
\hline \multicolumn{11}{|l|}{$\mathrm{ZPG}$} \\
\hline$x=1210 \mathrm{~mm}$ & 30.2 & 19.7 & 3.51 & 2.49 & 1.41 & 1.125 & 1439 & 4889 & 42 & 0.1 \\
\hline$x=1210 \mathrm{~mm}$ & 39.2 & 18.8 & 3.15 & 2.28 & 1.38 & 1.455 & 1776 & 5806 & 79 & 0.1 \\
\hline \multicolumn{11}{|l|}{ Schloemer } \\
\hline- & 43.6 & 25.6 & 5.26 & 3.33 & 1.58 & 1.30 & 2150 & 9180 & 1237 & 3.3 \\
\hline \multicolumn{11}{|c|}{ Catlett et $a l$. , open angle $=7^{\circ}$} \\
\hline$x=50 \mathrm{~mm}$ & 9.0 & 91.2 & 18.36 & 11.11 & 1.65 & 0.27 & 1616 & 6421 & 27 & 5.6 \\
\hline$x=204 \mathrm{~mm}$ & 18.1 & 73.1 & 14.36 & 9.27 & 1.55 & 0.55 & 2598 & 10775 & 75 & 3.0 \\
\hline$x=406 \mathrm{~mm}$ & 28.3 & 66.5 & 10.05 & 7.1 & 1.42 & 0.92 & 3992 & 12909 & 165 & 1.6 \\
\hline \multicolumn{11}{|c|}{ Open angle $=12^{\circ}$} \\
\hline$x=154 \mathrm{~mm}$ & 18.4 & 72.0 & 15.84 & 9.99 & 1.59 & 0.51 & 2376 & 11806 & 282 & 14.5 \\
\hline$x=210 \mathrm{~mm}$ & 28.1 & 68.5 & 11.96 & 8.28 & 1.44 & 0.87 & 3851 & 14947 & 625 & 8.4 \\
\hline \multicolumn{11}{|c|}{ Open angle $=17^{\circ}$} \\
\hline$x=106 \mathrm{~mm}$ & 28.4 & 73.1 & 16.34 & 10.1 & 1.62 & 0.71 & 3388 & 18429 & 900 & 24.3 \\
\hline \multicolumn{11}{|c|}{ Suryadi \& Herr, AOA $=-0.8^{\circ}$} \\
\hline$x / c=0.77$ & 53.3 & 6.1 & 1.22 & 0.78 & 1.56 & 1.95 & 772 & 2676 & 6209 & 1.7 \\
\hline$x / c=0.88$ & 49.6 & 8.0 & 1.72 & 1.06 & 1.63 & 1.54 & 803 & 3370 & 6574 & 4.0 \\
\hline$x / c=0.96$ & 45.9 & 10.3 & 2.60 & 1.44 & 1.80 & 1.10 & 731 & 4242 & 8206 & 14.9 \\
\hline \multicolumn{11}{|l|}{$\mathrm{AOA}=3.2^{\circ}$} \\
\hline$x / c=0.77$ & 54.0 & 7.4 & 1.65 & 0.99 & 1.66 & 1.77 & 851 & 3447 & 7724 & 3.4 \\
\hline$x / c=0.88$ & 49.4 & 10.1 & 2.56 & 1.42 & 1.81 & 1.26 & 821 & 4493 & 7059 & 9.6 \\
\hline$x / c=0.96$ & 45.7 & 13.2 & 4.17 & 1.94 & 2.15 & 0.77 & 658 & 5690 & 5949 & 35.3 \\
\hline \multicolumn{11}{|c|}{ Herrig et al., $\mathrm{AOA}=0^{\circ}$} \\
\hline$x / c=0.99$ & 33.4 & 13.5 & 2.77 & 1.64 & 1.69 & 0.99 & 863 & 3521 & 1762 & 4.2 \\
\hline \multicolumn{11}{|l|}{$\mathrm{AOA}=4^{\circ}$} \\
\hline$x / c=0.99$ & 62.7 & 12.4 & 3.68 & 2.03 & 1.82 & 1.50 & 1209 & 8166 & 6202 & 8.6 \\
\hline
\end{tabular}

ZPG cases.

Spectra from the RRM model present no clearly different slopes between medium and high frequencies, except for the cases for ZPG and very weak APG, e.g. Hu \& Herr AOA $=6^{\circ}, x=1128 \mathrm{~mm}$, where the spectra roll off at high frequencies with a much faster slope than the measured ones. The reason for that is the function $A_{2}=\min \left(3,19 / \sqrt{R_{T}}\right)+7$ in Eq. (2), which could result in a faster roll-off at high frequencies for small $R_{T}$. A poor prediction of the spectral slope at medium frequencies for the test case of Catlett et al. is shown in Fig. 2. For the test cases of $\mathrm{Hu} \&$ Herr, Catlett et al. and Suryadi \& Herr more than $5 \mathrm{~dB}$ discrepancy in peak level is found and the maximum discrepancy is about $12 \mathrm{~dB}$ found in test case of Suryadi \& Herr. Good agreement with the test cases of Schloemer and Herrig et al. is obtained.

The KBLWK model formulates a constant spectral slope at low, medium and high frequencies, only the extension of the mid-frequency range and the spectral amplitude are governed by boundary layer 
parameters. Therefore, a slope variation at medium frequencies due to APG effects shown in test cases of $\mathrm{Hu} \&$ Herr, Catlett et al. and Suryadi \& Herr can not be predicted and the slope at medium frequencies is too steep for ZPG cases. A good prediction of the peak amplitude is obtained except for the test case of Catlett et al., which shows a discrepancy of $10 \mathrm{~dB}$. The spectral peak location is well predicted for most test cases. Good agreement with measured spectra at positions in the vicinity of trailing edge is shown in Fig. 3(c,f) and Fig. 5.

The CFAS model underpredicts the spectral amplitude for all test cases except for the case of Catlett et al.. The discrepancy can be larger than $15 \mathrm{~dB}$. The trend of variation of the spectral slope at medium frequencies is not well predicted, e.g. a contradictory trend is shown in Fig. 1(a,f) and Fig. 3(a,f), where the slope at medium frequencies should be steeper due to a stronger APG.

$\mathrm{HH}$ model predicts well the spectral slope at medium frequencies and the roll-off frequency at high frequencies for test cases of Hu \& Herr, Catlett et al. and Suryadi \& Herr, except for one case with open angle $=17^{\circ}$ of Catlett et al., which may be caused by a boundary layer separation occurring upstream of the measurement position. A good prediction of the peak amplitude is obtained, except for the test case of Suryadi \& Herr, which is mainly due to the imprecisely predicted spectral peak location. A slope of $\omega$ at low frequencies used in this model shows a better agreement with the measured spectra than the other models which own an $\omega^{2}$ slope.

\section{New model}

The proposed model should be capable to predict the trend of the mid-frequency slope change due to APG effects, the extension of the mid-frequency range, the spectral peak amplitude and the peak location. From the previous discussion, it is shown that the HH model predicts well the slope change at medium frequencies using the boundary layer shape factor as driving parameters. Also good agreement of the spectral slope at low and high frequencies with the measured spectra is shown. The peak amplitude is well predicted except for one test case, which is mainly caused by an imprecise prediction of the peak location. Therefore, the new model will adopt the form of the HH model and aims to improve the prediction of the spectral peak location. Note that, the KBLWK model formulates a constant peak location in non-dimensional frequency domain and the prediction of the peak location shows good agreement with measured spectra.

Fig. 7 shows the scaled spectra from the test cases listed in Table 1. Configurations not included in this plot are: 1, spectra measured in the vicinity of the trailing edge, i.e. measurements from Suryadi \& Herr at $x / c=0.96$ and from Herrig et al.. For these configurations spectra can be impacted by the trailing edge scatter effect. Furthermore, the boundary layer parameter provided by XFOIL calculations can be imprecise in the vicinity of the trailing edge. ${ }^{49}$, the measured position located not far downstream of a boundary layer separation, i.e. measurements from Catlett et al. with open angle of $17^{\circ}$.

An noteworthy finding from Fig. 7 is that the scaled spectra can be divided into three groups. Group I for the cases of $\mathrm{Hu} \&$ Herr and Schloemer, APG boundary layers developed at a flat plate with airfoils mounted above, $18.8 \mathrm{~mm} \leq \delta \leq 35.0 \mathrm{~mm}$; group II for the case of Catlett et al., APG boundary layers developed at a tapered trailing edge section of a flat plate, $66.5 \mathrm{~mm} \leq \delta \leq 91.2 \mathrm{~mm}$; group III for the case of Suryadi \& Herr, APG boundary layers developed on the airfoil suction side, $6.1 \mathrm{~mm} \leq \delta \leq 13.2 \mathrm{~mm}$. The spectral peaks of each group collapse well by itself, which may indicate a good scaling of the spectral peak is given when boundary layers experience a similar development history or the boundary layer thicknesses have the same order. Although a good peak collapse is shown in each test case by itself, the differences in peak amplitude are still about $10 \mathrm{~dB}$ between different test configurations.

Nevertheless, the spectral peak location is located in a small range between $\omega \theta / U_{0}$ of $0.2-0.35$. Based on the good prediction of the peak location from the KBLWK model with a constant for predicting the peak location, a constant value for the variable $d$ in the denominator in Eq. (5) is searched, with which the peak can be located in the range between $\omega \theta / U_{0}$ of $0.2-0.35$, and a value of 0.07 is found. The spectral amplitude in Eq. (5) is nearly independent on the choice of the value of $d$ at higher frequencies. Therefore, the amplitude function can keep the form as it is. Thus, the model is rewritten as

$$
\frac{\Phi(\omega) u_{\tau}}{Q^{2} \theta}=\frac{(81.004 d+2.154) \cdot 10^{-7} \cdot\left(\omega \theta / U_{0}\right)}{\left[\left(\omega \theta / U_{0}\right)^{1.5 h^{1.6}}+0.07\right]^{1.13 / h^{0.6}}+\left[7.645 R e_{\tau}^{-0.411} \cdot\left(\omega \theta / U_{0}\right)\right]^{6}},
$$

where $\log _{10}(d)=-5.8 \cdot 10^{-5} \cdot R e_{\theta} H-0.35$ and $h=1.169 \ln (H)+0.642$. Note that, a function of boundary layer shape factor $H$ and momentum Reynolds number $R e_{\theta}$ is used in this expression to compensate the difference of peak amplitude between each test cases, i.e if the spectral peak collapses well for each test case, a constant can be placed herein instead of the function of $H$ and $R_{\theta}$.

A good collapse of the spectral peak is found using $u_{\tau} / Q^{2} l_{\text {ref }}$ as the pressure scaling parameter, 
shown in Fig. 8(a), where $l_{\text {ref }}=1 \mathrm{~m}$ served as a reference length instead of the common boundary layer based length scale. The scaling parameter is mainly driven by outer velocities, and a single boundary layer related parameter $u_{\tau}$ used in this scaling parameter seems to be able to reflect effects of the APG on the spectral peak level. Based on the well scaled spectra from the different configurations, the function of $H$ and $R e_{\theta}$ used in Eq. (6) for adjusting the spectral amplitude can be replaced by a constant, namely a value of $3.1 \cdot 10^{-9}$ is found to achieve good agreement with the measured spectra. A new expression of the model is written as

$$
\frac{\Phi(\omega) u_{\tau}}{Q^{2} l_{\text {ref }}}=\frac{3.1 \cdot 10^{-9} \cdot\left(\omega \theta / U_{0}\right)}{\left[\left(\omega \theta / U_{0}\right)^{1.5 h^{1.6}}+0.07\right]^{1.13 / h^{0.6}}+\left[7.645 R e_{\tau}^{-0.411} \cdot\left(\omega \theta / U_{0}\right)\right]^{6}},
$$

where $l_{\text {ref }}=1 \mathrm{~m}$ and $h=1.169 \ln (H)+0.642$. Fig. 8(b) shows spectra from Eq. (7) for three different boundary layer shape factors at Reynolds number $R e_{\tau}=1500$. Peak amplitude and location of the formulated spectra are in good agreement with the measured spectra, see comparison between Fig. 8(a) and Fig. 8(b).

Figs. 9-13 show predictions from the new model with two different expressions from Eq. (6) and Eq. (7) for the APG test cases, and Fig. 14 for the ZPG cases. Both expressions of the new model show good agreement with the measured spectra. The spectral slope over the whole frequency range is well predicted. Exceptions are the slope at medium and high frequencies for measurements from Schloemer and Herrig et al.. For these measurements, flush-mounted sensors with diameters of $1.5 \mathrm{~mm}$ and $1.6 \mathrm{~mm}$ were used to measure the surface pressure fluctuations, which cause an attenuation in spectral amplitude at medium and high frequencies due to the large sensor size. Although the measured spectra were corrected using Corcos correction, ${ }^{50}$ uncertainties at higher frequencies could still be caused, which may explain the difference at higher frequencies between the prediction and those measurements. Corcos correction assumed a uniform sensitivity for the sensors, whereas an actual sensor has a deflective sensitivity, e.g. for a condenser microphone the sensitivity has the maximum at the center and decreases near the edge. Blake ${ }^{17}$ showed that the measured acceptance of a condenser microphone at higher wavenumber domain could be more than $5 \mathrm{~dB}$ smaller than the calculated theoretical acceptance with a uniform sensitivity. The difference is noticeable from $\omega U_{c} / r>1$. This discrepancy at the acceptance will cause a smaller amplitude at higher frequencies even after using Corcos correction. A new result (not yet published) for the wall pressure spectra measured by flush-mounted $2.54 \mathrm{~mm}$ diameter Kulite sensors with so called B-screen for ZPG boundary layers using an almost identical experimental setup as ${ }^{31}$ shows an under-correction using Corcos correction from about $\omega U_{c} / r>0.5$. However, a different conclusion for the Corcos correction was made from Lueptow, ${ }^{51}$ who argued that the Corcos correction over-corrects the wall spectra at higher frequencies. The reason for the different results is because different acceptances of the sensors were used. The one Lueptow applied is given by Smol'yakov and Tkachenko, ${ }^{52}$ who assumed an idealized deflection sensor and described its sensitivity distribution with the first vibration mode of the membrane with a Bessel function. The formulated acceptance has a larger value at higher wavenumber domain than the one with an uniform sensitivity. Another issue should be also considered when using the Corcos correction is that the convective velocity is much slower for an APG boundary layer than for a ZPG boundary. To the author, it makes more sense to use the phase velocity for the closest distance (the order of sensor size). The convective velocity $U_{c}(\omega)$ for an APG boundary layer at the closest distance could be less than $0.3 U_{0}$ at higher frequencies, ${ }^{31}$ which is much slower than the usually used $0.6-0.8 U_{0}$. The too large convective velocity used in the Corcos-correction will lead to a under-correction for the wall spectra. Furthermore, the APG increase the turbulence decay in streamwise compared to the ZPG. A larger turbulence decay can further increase the attenuation due to the finite sensor size. Besides the sensor effect, as discussed previously, the trailing edge scatter effect and a possible inaccuracy of the boundary layer parameters in the vicinity of trailing edge provided by XFOIL calculation could produce the prediction uncertainty and increase the discrepancy compared to the measured spectra. This issue affects the results for the case of Herrig et al. and the case of Suryadi \& Herr at $x / c=0.96$, and the discrepancy is larger at a larger AOA. A poor prediction is made for the case of Catlett et al. with an open angle of $17^{\circ}$, which is probably due to a separated boundary layer occurred near upstream of the measured location. In addition, a good prediction is also obtained for the ZPG boundary layers.

\section{Conclusion}

An empirical spectral model of wall pressure fluctuations for adverse and zero pressure gradient boundary layers is proposed in this work. Experimental data which were acquired by different experimental configurations covering a large Reynolds number range are selected to develop the new model. Predictions of existing models for adverse pressure gradient boundary layers to the selected database 
are shown and results between the models are discussed. Three major changes of the Hu \& Herr model were made compared to the other models: 1 , the boundary layer shape factor instead of Clauser's equilibrium parameter is used to predict the spectral slope variation at medium frequencies due to adverse pressure gradient effects. 2, the pressure scaling parameter for adverse pressure gradient boundary layers is mainly driven by dynamic pressure, i.e. the local free-stream velocity, instead of the wall shear stress. 3 , an $\omega^{1}$ slope at low frequencies is applied based on an average slope of the measured spectra from different configurations for adverse pressure gradient boundary layers, whereas an $\omega^{2}$ slope is applied by the others.

Based on the principally good performance of the $\mathrm{Hu} \&$ Herr model model, its basis form is adopted to develop the present model. A major effort made in developing the present model is to improve the prediction of the spectral peak location. Due to the good result for predicting the peak location by the Kamruzzaman et al. model using a constant value, a constant instead of a function of the momentum Reynolds number and the boundary layer shape factor in the denominator in Eq. (5) for adjusting the peak location is applied. For prediction of the spectral amplitude, two approaches are applied. The first one (Eq. (6)) uses the formulation of the Hu \& Herr model to adjust the amplitude with a function of the momentum Reynolds number and the boundary layer shape factor. The second one (Eq. (7)) takes advantage of a new pressure scaling parameter, which allows the spectral peak to collapse within about $4 \mathrm{~dB}$ for different experimental configurations. Therefore, a constant value for controlling the spectral amplitude can be applied.

Results of the present model with both expressions are compared to measured spectra from the database. Very good agreement is obtained, except for some specific configurations. These are: 1, spectra measured in the vicinity of a trailing edge. Reason for that could be the trailing edge scattering effect and possible imprecise estimations of input boundary layer parameters in the trailing edge area, where not available from the measurements. These are provided by means of XFOIL calculations in the current study. 2, spectra measured at positions not far away downstream from a boundary-layer separation. Uncertainty of $\pm 2 \mathrm{~dB}$ compared to the experimental database excluding the mentioned specific cases is shown using expression Eq. (7), the uncertainty is larger for expression Eq. (6), which is about $\pm 4 \mathrm{~dB}$. Good agreement for zero pressure gradient boundary layers from the present model is also obtained.

\section{Acknowledgments}

This work was conducted in the framework of the DLR project CENT.

\section{References}

${ }^{1}$ Kraichnan, R. H., "Pressure fluctuations in turbublent flow over a flat plate." J. Acoust. Soc. Am., Vol. 28(3), 1956, pp. $378-390$

${ }^{2}$ Willmarth, W. W. and Wooldridge, C. E., "Measurements of the fluctuating pressure at the wall beneath a thick turbulent boundary layer." J. Fluid Mech., Vol. 14, 1962, pp. 187-210.

${ }^{3}$ Corcos, G. M., "The structure of the turbulent pressure field in boundary layer flows." J. Fluid Mech., Vol. 18, 1964, pp. 353-378.

${ }^{4}$ Bull, M. K., "Wall pressure fluctuations associated with subsonic turbulent boundary layer flow." J. Fluid Mech., Vol. 28, 1967, pp. 719-754.

${ }^{5}$ Blake, W. K., "Turbulent boundary layer wall pressure fluctuations on smooth or rough walls." J. Fluid Mech., Vol. 44(4), 1970, pp. 637-660.

${ }^{6}$ Panton, R. L. and Linebarger, J. H., "Wall pressure spectra for equilibrium boundary layers." J. Fluid Mech., Vol. 65, 1974, pp. 261-287.

${ }^{7}$ Chase, D. M., "Modeling the wave-vector frequency spectrum of turbulent boundary layer wall pressure." J. Sound Vib., Vol. 70, 1980, pp. 29-68.

${ }^{8}$ Spalart, P. R., "Direct simulation of a turbulent boundary layer up to $\operatorname{Re}_{\theta}=1410 . "$ J. Fluid Mech., Vol. 187, 1988, pp. 61-98.

${ }^{9} \mathrm{Kim}$, J., "On the structure of pressure fluctuations in simulated turbulent channel flow." J. Fluid Mech., Vol. 205, 1989, pp. 421-451.

${ }^{10}$ Farabee, T. M. and Casarella, M. J., "Spectral features of wall pressure fluctuations beneath turbulent boundary layers." Phys. Fluids, Vol. A3(10), 1991, pp. 2410-2420.

${ }^{11}$ Chang, P., Piomelli, U., and Blake, W. K., "Relationship between wall pressure and velocity-field sources." Phys. Fluids, Vol. 11, 1999, pp. 3434-3448.

${ }^{12}$ Viazzo, S., Dejoan, A., and R.Schiestel, "Spectral features of the wall-pressure fluctuations in turbulent wall flows with and without perturbations using LES." J. Heat and Fluid Flow, Vol. 22, 2001, pp. 39-52.

${ }^{13}$ Leclercq, D. J. J. and Bohineust, X., "Modeling the wave-vector frequency spectrum of turbulent boundary layer wall pressure." J. Sound Vib., Vol. 257(3), 2002, pp. 477-501.

${ }^{14}$ Arguillat, B., Ricot, D., Robert, G., and Bailly, C., "Measurements of wavenumber-frequency spectrum of wall pressure fluctuations under turbulent flows." AIAA Paper, 2005. 
${ }^{15}$ Ehrenfried, K. and Koop, L., "Experimental study of pressure fluctuations beneath a compressible turbulent boundary layer." AIAA Paper, 2008.

${ }^{16}$ Gloefelt, X. and Berland, J., "Turbulent boundary layer wall pressure fluctuations on smooth or rough walls." J. Fluid Mech., Vol. 723, 2013, pp. 318-351.

${ }^{17}$ Blake, W. K., Mechanics of flow-induced sound and vibration, Academic Press, Inc., 1986.

${ }^{18}$ Efimtsov, B., "Characteristics of the field of turbulent wall pressure fluctuations at large Reynolds numbers." Sov. Phys. Acoust, Vol. 28, 1982, pp. 289-292.

${ }^{19}$ Efimtsov, B., "Similarity criteria for the spectra of wall pressure fluctuations in a turbulent boundary layer." Sov. Phys. Acoust, Vol. 30, 1984, pp. 33-35.

${ }^{20}$ Howe, M., Acoustics of fluid-structure interactions, Cambridge University Press, 1998.

${ }^{21}$ Smol'yakov, A., "Calculation of the spectra of pseudosound wall-pressure fluctuations in turbulent boundary layers." Acoustical Physics, Vol. 3, 2000, pp. 342-347.

${ }^{22}$ Goody, M., "Empirical spectral model of surface pressure fluctuations." AIAA Journal, Vol. 42, No.9, 2004.

${ }^{23}$ Schloemer, H. H., "Effects of pressure gradients on turbulent-boundary-Layer wall-pressure fluctuations." J. Acoust. Soc. Am., Vol. 42(1), 1967, pp. 93-113.

${ }^{24}$ Burton, T. E., "Wall pressure fluctuations at smooth and rough surfaces under turbulent boundary layers with favorable and adverse pressure gradients." Tech. rep., Acoustics and vibration Lab. Massachusetts Inst. of Technology, 1973.

${ }^{25}$ Blake, W. K., "A statistical description of pressure and velocity fields at trailing edges of a flat strut." Tech. rep., David W. Taylor Naval Ship Research and Development Center, 1975.

${ }^{26}$ Simpson, R., Ghodbane, M., and McGrath, B., "Surface pressure fluctuations in a separation turbulent boundary layer." J. Fluid Mech., Vol. 177(1), 1987, pp. 167-186.

${ }^{27}$ Herrig, A., Validation and application of a hot-wire based method for trailing edge noise measurements on airfoils., Ph.D. thesis, Iniversity of Stuttgart, 2012.

${ }^{28}$ Catlett, M. R., Forest, J. B., Anderson, J. M., and Stewart, D. O., "Empirical spectral model of surface pressure fluctuations beneath adverse pressure gradients." AIA A Paper, 2014.

${ }^{29}$ Salze, E., Bailly, C., Marsden, O., Jondeau, E., and Juvé, D., "An experimental characterisation of wall pressure wavevector-frequency spectra in the presence of pressure gradients." AIAA Paper, 2014.

${ }^{30}$ Suryadi, A. and Herr, M., "Wall pressure spectra on a DU96-W-180 profile from low to pre-stall angles of attack." AIA A Paper, 2015.

${ }^{31} \mathrm{Hu}, \mathrm{N}$. and Herr, M., "Characteristics of wall pressure fluctuations for a flat plate turbulent boundary layer with pressure gradients." AIA A Paper, 2016.

${ }^{32}$ Rozenberg, Y., Robert, G., and Moreau, S., "Wall-pressure spectral model including the adverse pressure gradient effects." AIA A Journal, Vol. 50(10), 2012, pp. 2168-2179.

${ }^{33}$ Clauser, F. H., "Turbulent boundary layers in adverse pressure gradients." Journal of the Aeronautical Sciences, Vol. 21(2), 1954, pp. 91-108.

${ }^{34}$ Kamruzzaman, M., Bekiropoulos, D., Lutz, T., and Würz, W., "A semi-empirical surface pressure spectrum model for airfoil trailing-edge noise prediciton." International journal of aeroacoustics, Vol. 14, 2015.

${ }^{35}$ Wolf, A., Kamruzzaman, M., Würz, W., Lutz, T., and Krämer, E., "Wall pressure fluctuation (WPF) and trailingedge noise measurements on a NACA64-418 airfoil." Tech. rep., Institute of Aerodynamics and Gas Dynamics, University of Stuttgart, 2009.

${ }^{36}$ Rozenberg, Y., Modélisation analytique du bruit aérodynamique à large bande des machines tournantes: utilisation de calculs moyennés de mécanique des fluides., Ph.D. thesis, L' École Centrale de Lyon, 2007.

${ }^{37}$ Bertagnolio, F., "Boundary layer measurements of the NACA0015 and implications for noise modeling." Tech. rep., RISOE DTU, National Laboratory for Sustainable Energy, 2011.

${ }^{38}$ Garcia-Sagrado, A. and Hynes, T., "Stochastic estimation of flow near the trailing edge of a NACA0012 airfoil." Experiments in Fluids, Vol. 51, 2011, pp. 1057-1071.

${ }^{39}$ Brooks, T. F. and Hodgson, T. H., "Trailing edge noise prediction from measured surface pressures." J. Sound Vib., Vol. 78(1), 1981, pp. 69-117. 253.

${ }^{40}$ Mellor, G. L. and Gibson, D. M., "Equilibrium turbulent boundary layers." J. Fluid Mech., Vol. 24(2), 1966, pp. 225-

${ }^{41}$ Cole, D., "The law of the wake in the turbulent boundary layer." J. Fluid Mech., Vol. 1(2), 1956, pp. 191-226.

${ }^{42}$ White, F. M., Viscous fluid flow, McGraw-Hill, 1974.

${ }^{43}$ Catlett, M. R., Anderson, J. M., Forest, J. B., and Stewart, D. O., "Empirical modeling of pressure spectra in adverse pressure gradient turbulent boundary layers." AIAA Journal, Vol. 54(2), 2016.

${ }^{44} \mathrm{Hu}$, N., Appel, C., Herr, M., Reiche, N., and Ewert, R., "Numerical study of Wall pressure fluctuations for zero and non-zero pressure gradient turbulent boundary layers." AIA A Paper, 2016.

${ }^{45}$ Hodgson, T. H., Pressure fluctuations in shear flow turbulence, Ph.D. thesis, University of London, 1962.

${ }^{46}$ Chang, P. A., "Wavenumber-frequency characteristics of partial wall pressures from large eddy simulations of a turbulent channel flow." Tech. rep., Naval Surface Warfare Center, 2001.

${ }^{47} \mathrm{Hu}, \mathrm{N}$., Reiche, N., and Ewert, R., "Simulation of turbulent boundary layer wall pressure fluctuations via Poisson equation and synthetic turbulence." (submitted to J. Fluid Mech., 2016).

${ }^{48} \mathrm{Hu}, \mathrm{N}$., Reiche, N., and Ewert, R., "Numerical investigation of wall pressure fluctuations for zero and adverse pressure gradient turbulent boundary layers using synthetic anisotropic turbulence." AIA A Paper, 2017.

${ }^{49}$ Suryadi, A., Martens, S., and Herr, M., "Trailing-edge noise reduction technologies for applications in wind energy." AIA A Paper, 2017.

${ }^{50}$ Corcos, G. M., "Resolution of pressure in turbulence." J. Acoust. Soc. Am., Vol. 35, 1964, pp. 192-199. 1995.

${ }^{51}$ Lueptow, R. M., "Transducer resolution and the turbulent wall pressure spectrum." J. Acoust. Soc. Am., Vol. 97 (1),

${ }^{52}$ Smol'yakov, A. V. and Tkachenko, V. M., The measurement of turbulent fluctuations: an introduction to hot-wire anemometry and related transducers., Springer-Verlag, Berlin Heidelberg New York, 1983. 


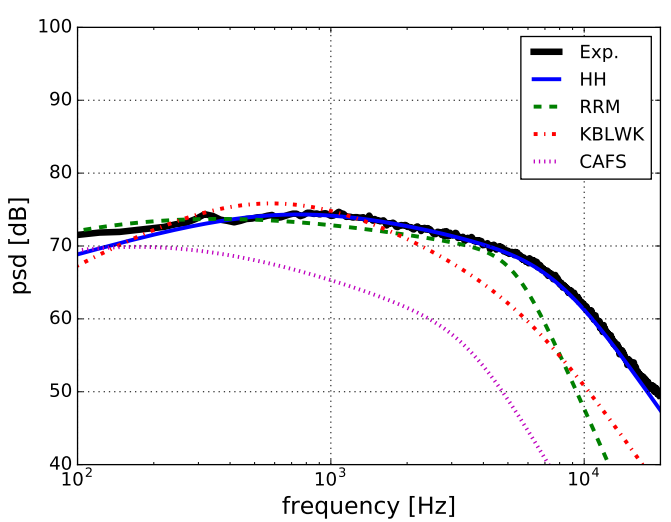

(a)

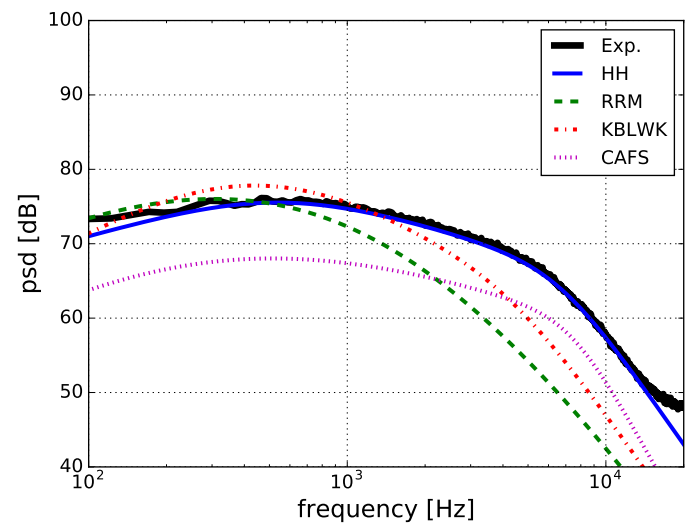

(c)

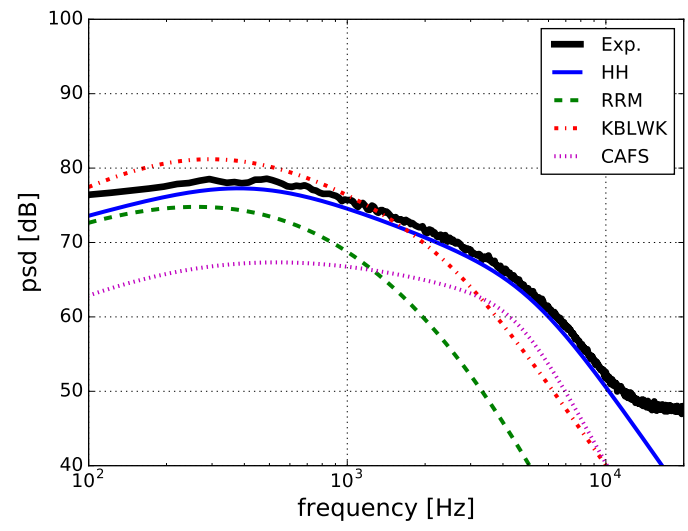

(e)

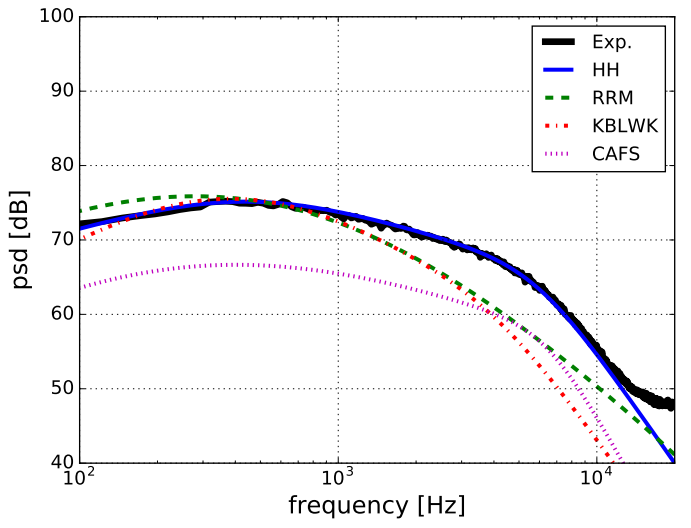

(b)

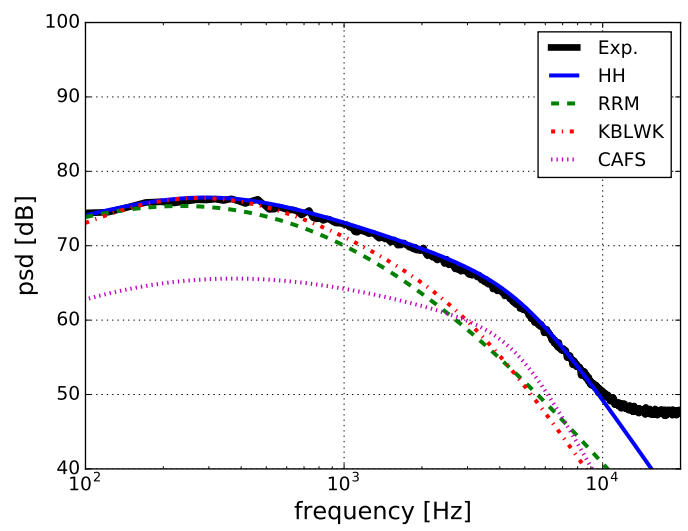

(d)

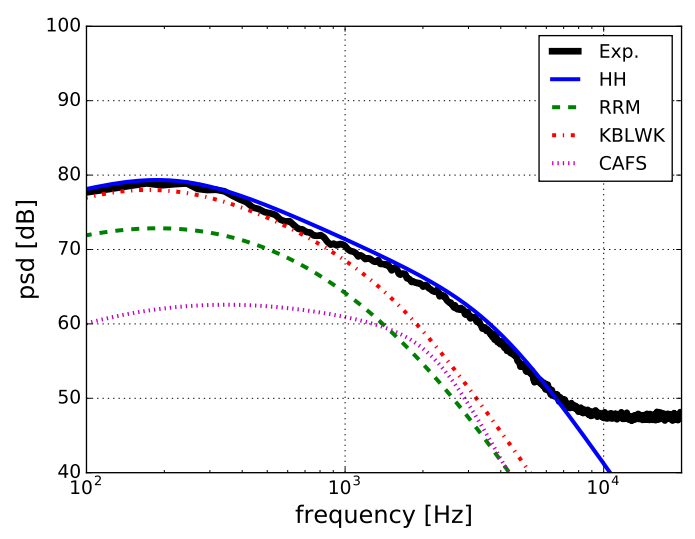

(f)

Figure 1: Predictions of different models for test case of Hu \& Herr; (a) $\mathrm{AOA}=6^{\circ}, U_{0}=32.3 \mathrm{~m} / \mathrm{s}$, distance of leading edge $x=1128 \mathrm{~mm}$; (b) AOA $=6^{\circ}, U_{0}=30.8 \mathrm{~m} / \mathrm{s}$, distance of leading edge $x=1210 \mathrm{~mm}$; (c) $\mathrm{AOA}=10^{\circ}, U_{0}=32.0 \mathrm{~m} / \mathrm{s}$, distance of leading edge $x=1128 \mathrm{~mm}$; (d) $\mathrm{AOA}=10^{\circ}, U_{0}=30.4 \mathrm{~m} / \mathrm{s}$, distance of leading edge $x=1210 \mathrm{~mm}$; (e) AOA $=14^{\circ}, U_{0}=31.2 \mathrm{~m} / \mathrm{s}$, distance of leading edge $x=1128 \mathrm{~mm}$; (f) $\mathrm{AOA}=14^{\circ}, U_{0}=29.9 \mathrm{~m} / \mathrm{s}$, distance of leading edge $x=1210 \mathrm{~mm}$. 


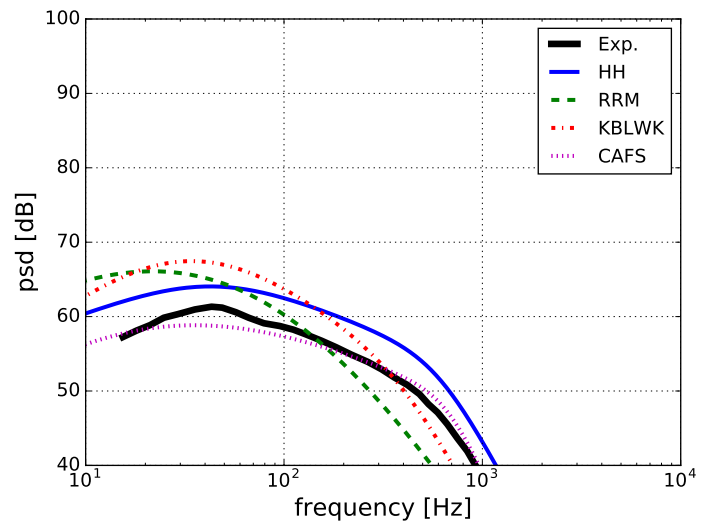

(a)

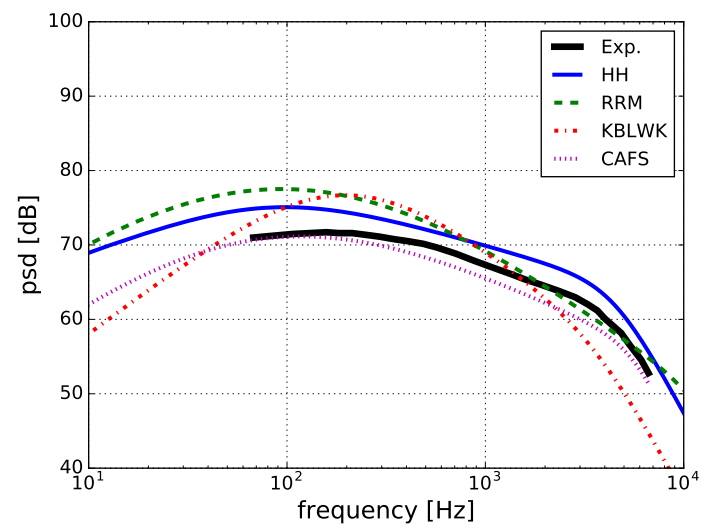

(c)

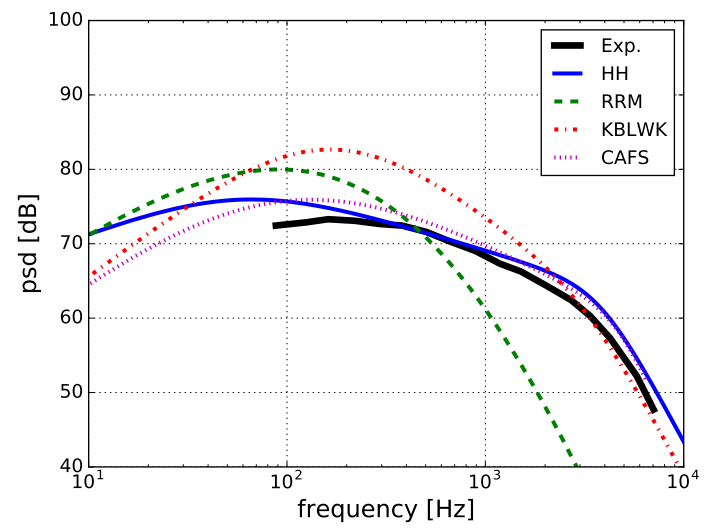

(e)

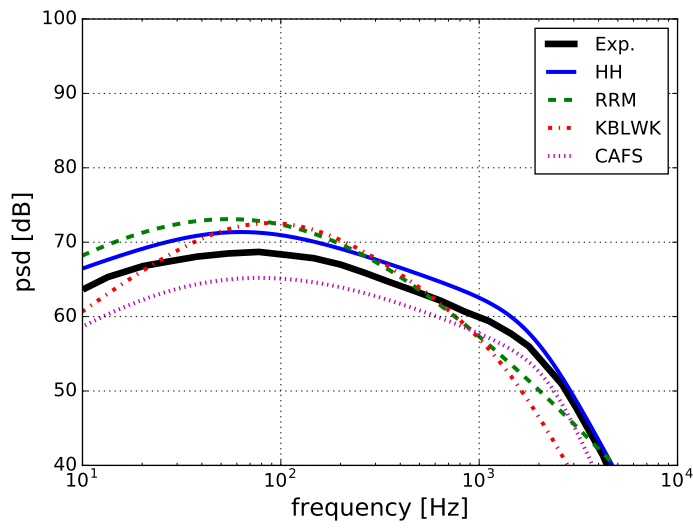

(b)

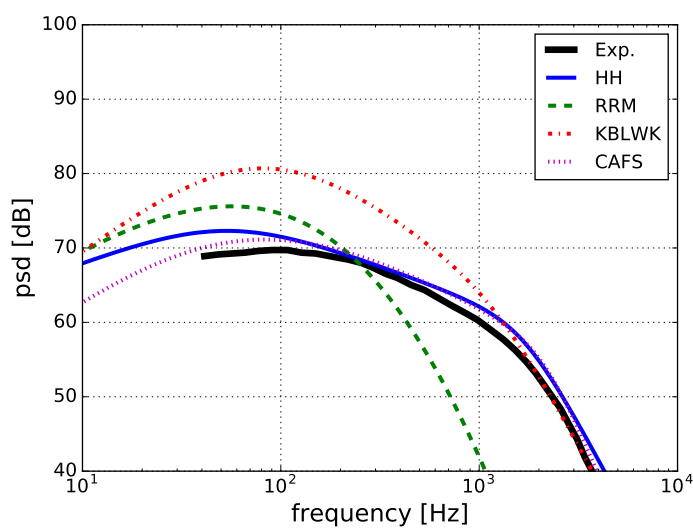

(d)

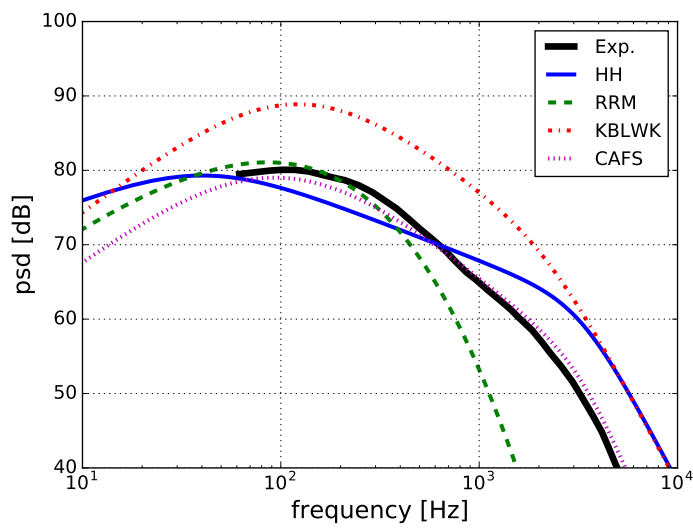

(f)

Figure 2: Predictions of different models for test case of Catlett et al.; (a) open angle $=7^{\circ}, U_{0}=9.0 \mathrm{~m} / \mathrm{s}$, distance of trailing edge $x=50 \mathrm{~mm}$; (b) open angle $=7^{\circ}, U_{0}=18.1 \mathrm{~m} / \mathrm{s}$, distance of trailing edge $x=204 \mathrm{~mm}$; (c) open angle $=7^{\circ}, U_{0}=28.3 \mathrm{~m} / \mathrm{s}$, distance of trailing edge $x=406 \mathrm{~mm}$; (d) open angle $=12^{\circ}, U_{0}=18.4 \mathrm{~m} / \mathrm{s}$, distance of trailing edge $x=154 \mathrm{~mm} ;(\mathrm{e})$ open angle $=12^{\circ}, U_{0}=28.1 \mathrm{~m} / \mathrm{s}$, distance of trailing edge $x=210 \mathrm{~mm}$; (f) open angle $=17^{\circ}, U_{0}=28.4 \mathrm{~m} / \mathrm{s}$, distance of trailing edge $x=106 \mathrm{~mm}$. 


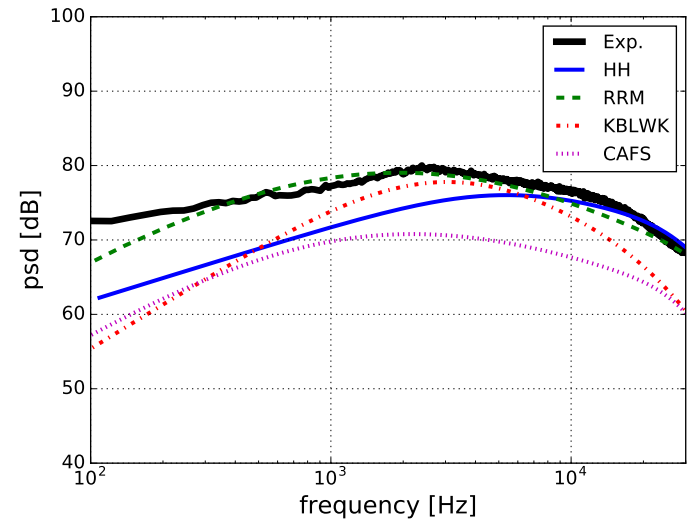

(a)

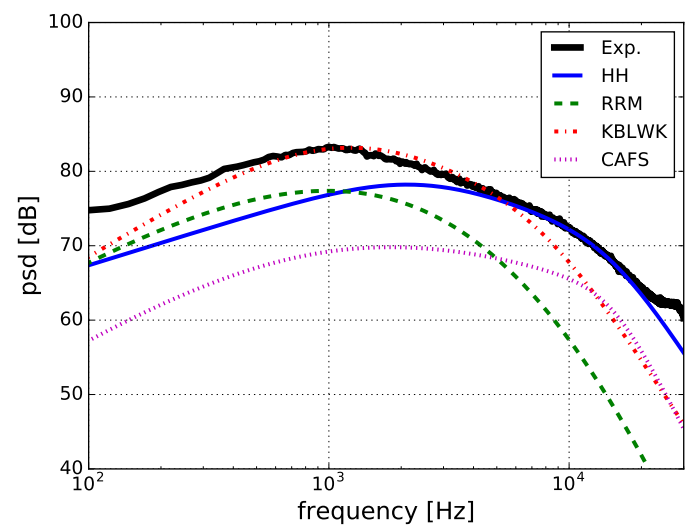

(c)

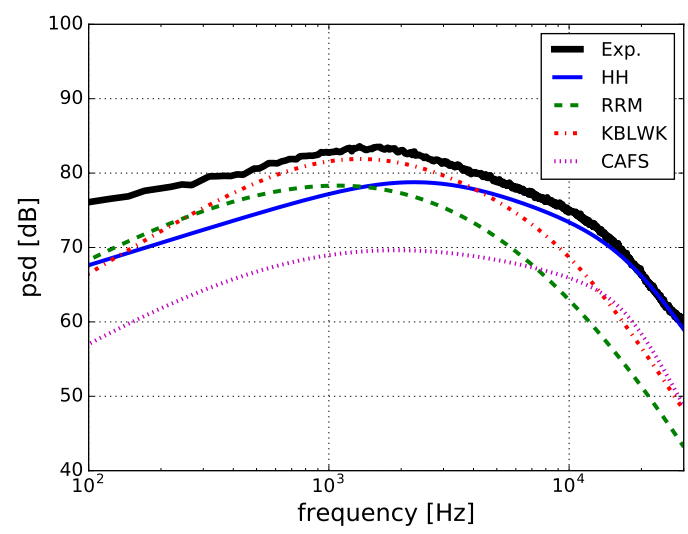

(e)

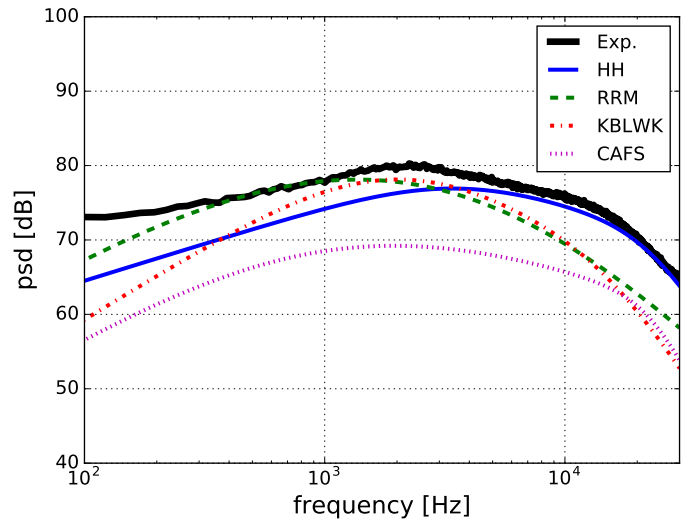

(b)

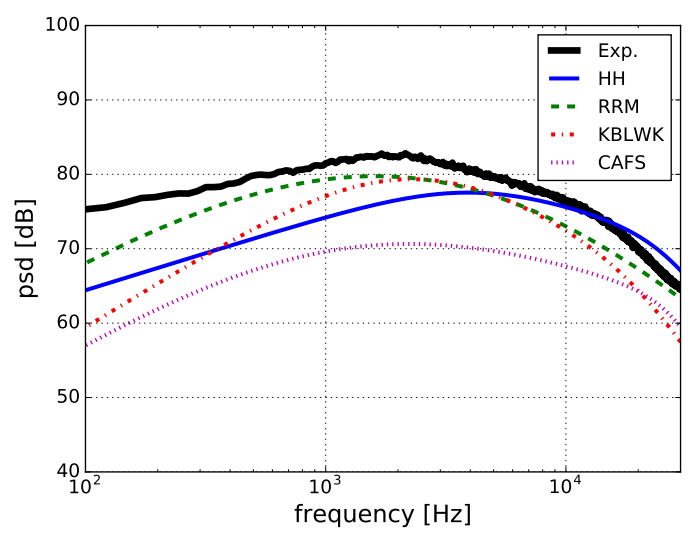

(d)

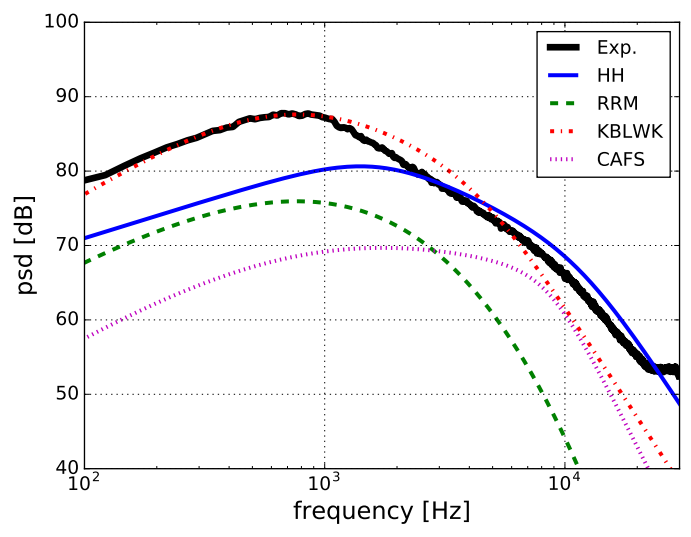

(f)

Figure 3: Predictions of different models for test case of Suryadi \& Herr,; (a) AOA $=-0.8^{\circ}, U_{0}=53.3 \mathrm{~m} / \mathrm{s}$, chord position $x / c=0.77$; (b) $\mathrm{AOA}=-0.8^{\circ}, U_{0}=49.6 \mathrm{~m} / \mathrm{s}$, chord position $x / c=0.88$; (c) $\mathrm{AOA}=-0.8^{\circ}$, $U_{0}=45.9 \mathrm{~m} / \mathrm{s}$, chord position $x / c=0.96 ;$ (d) $\mathrm{AOA}=3.2^{\circ}, U_{0}=54.0 \mathrm{~m} / \mathrm{s}$, chord position $x / c=0.77$; (e) $\mathrm{AOA}=3.2^{\circ}, U_{0}=49.4 \mathrm{~m} / \mathrm{s}$, chord position $x / c=0.88$; (f) $\mathrm{AOA}=3.2^{\circ}, U_{0}=45.7 \mathrm{~m} / \mathrm{s}$, chord position $x / c=0.96$. 


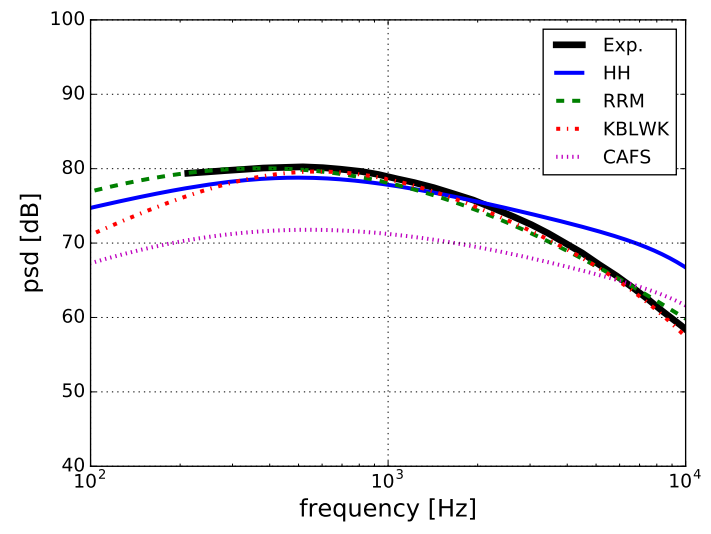

(a)

Figure 4: Predictions of different models for test case of Schloemer, $U_{0}=43.6 \mathrm{~m} / \mathrm{s}$.

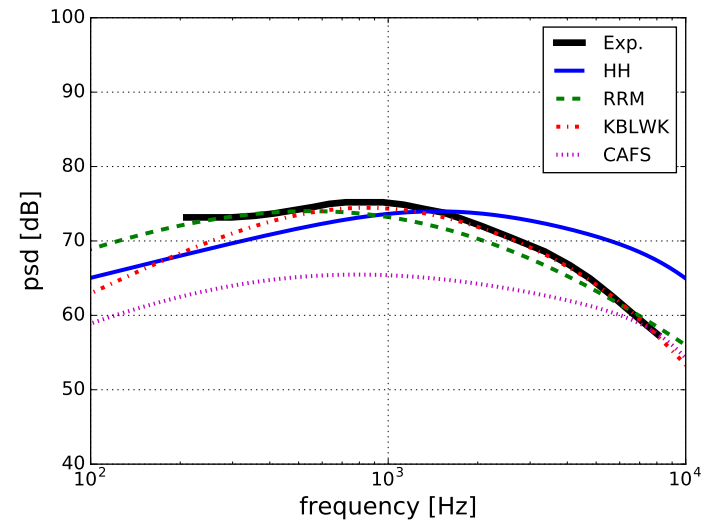

(a)

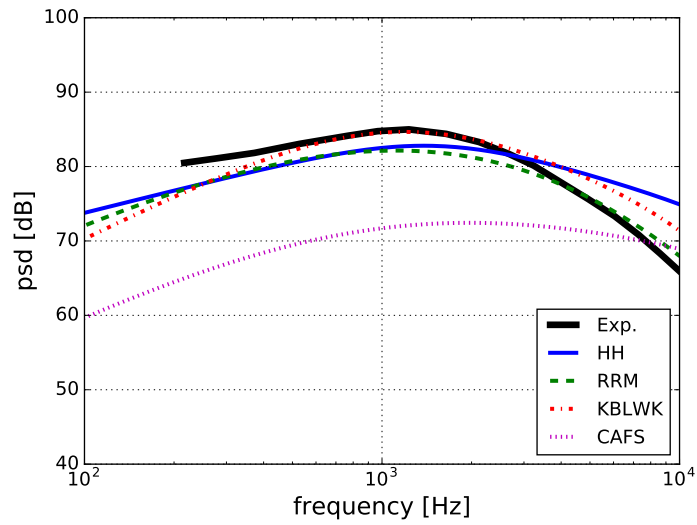

(b)

Figure 5: Predictions of different models for test case of Herrig et al.; (a) $\mathrm{AOA}=0^{\circ}, U_{0}=33.4 \mathrm{~m} / \mathrm{s}$, chord position $x / c=0.99$; (b) $\mathrm{AOA}=4^{\circ}, U_{0}=62.7 \mathrm{~m} / \mathrm{s}$, chord position $x / c=0.99$.

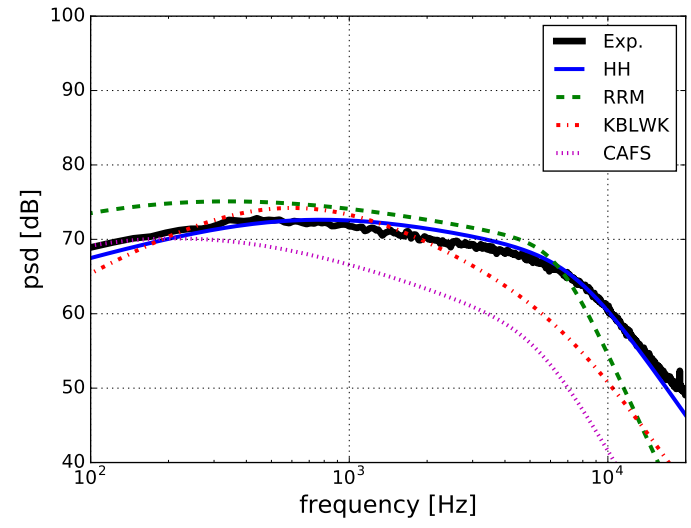

(a)

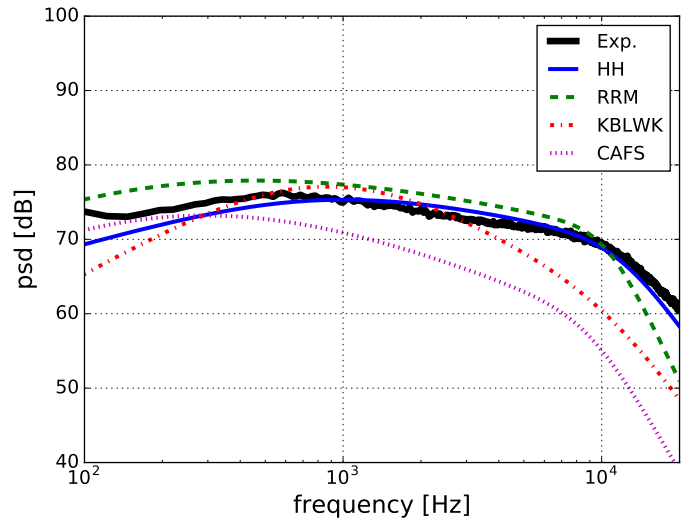

(b)

Figure 6: Predictions of different models for test case of Hu \& Herr for ZPG; (a) $U_{0}=30.2 \mathrm{~m} / \mathrm{s}$, distance of leading edge $x=1210 \mathrm{~mm}$; (b) $U_{0}=39.2 \mathrm{~m} / \mathrm{s}$, distance of leading edge $x=1210 \mathrm{~mm}$. 


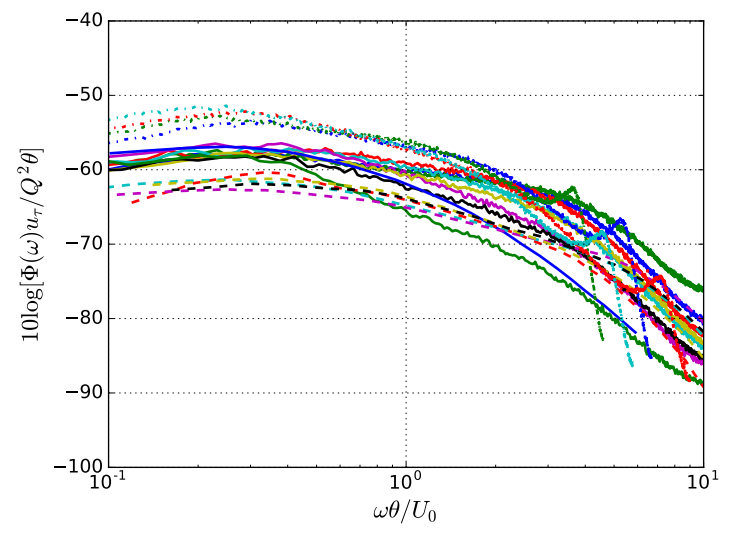

(a)

Figure 7: Spectra scaled by $u_{\tau} / Q^{2} \theta$ and $\theta / U_{0}$ from test cases of $\mathrm{Hu} \&$ Herr, Schloemer (-), Catlett et al. (- -) and Suryadi \& Herr (-.).

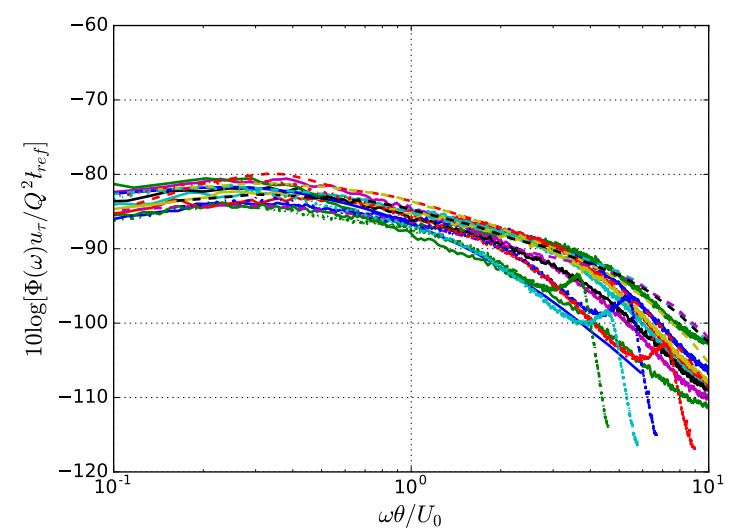

(a)

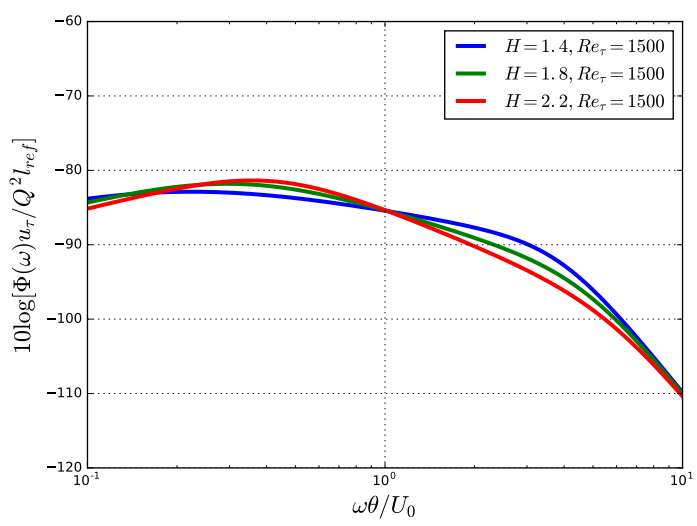

(b)

Figure 8: (a) Spectra scaled by $u_{\tau} / Q^{2} l_{\text {ref }}$ and $\theta / U_{0}$ from test cases of Hu \& Herr, Schloemer (-), Catlett et al. (- -) and Suryadi \& Herr (-.); (b) spectra from Eq. (7) with different boundary layer shape factors. 


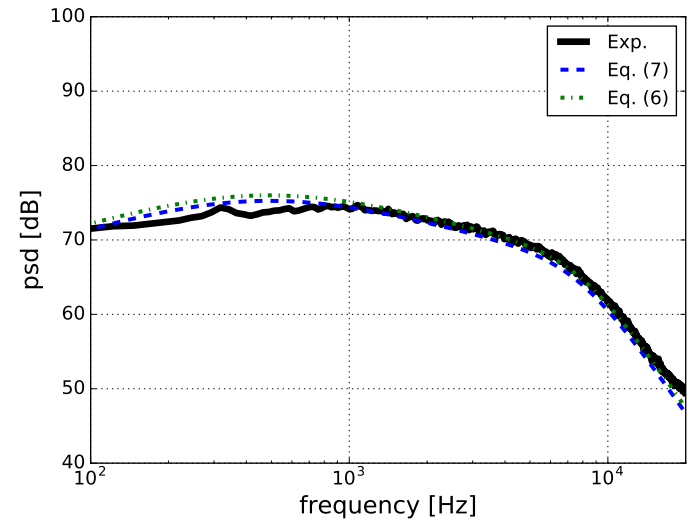

(a)

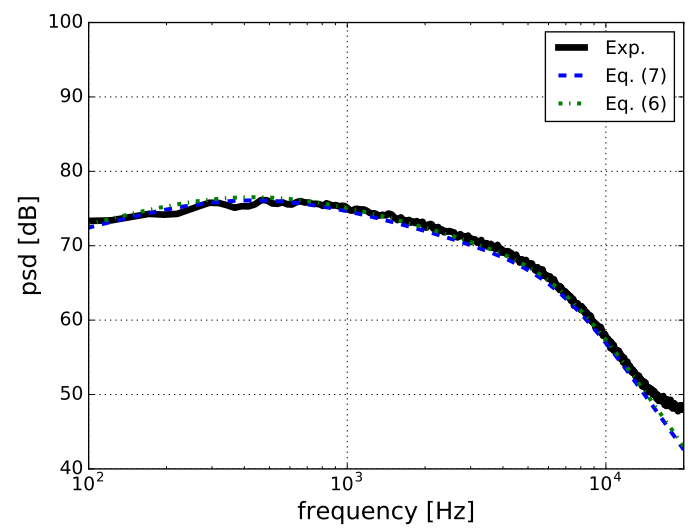

(c)

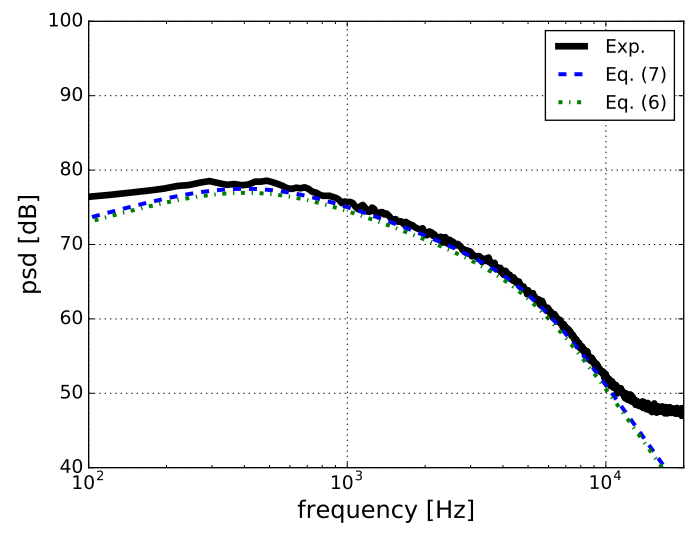

(e)

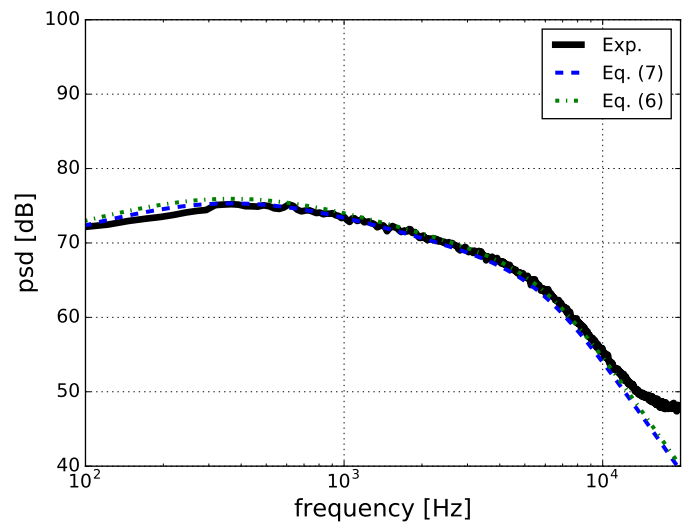

(b)

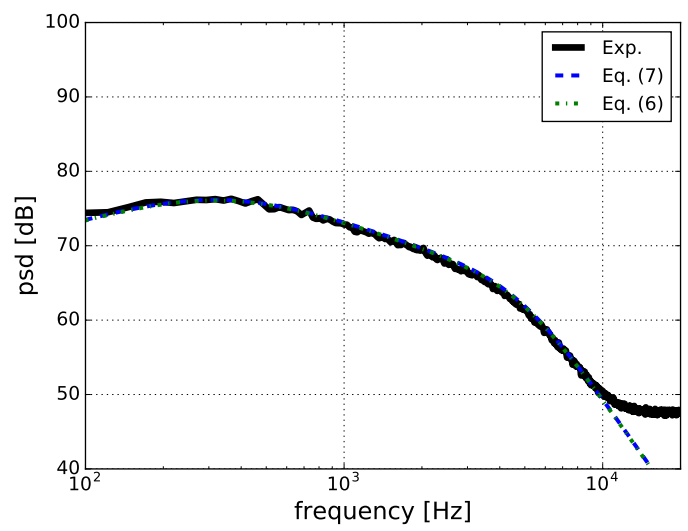

(d)

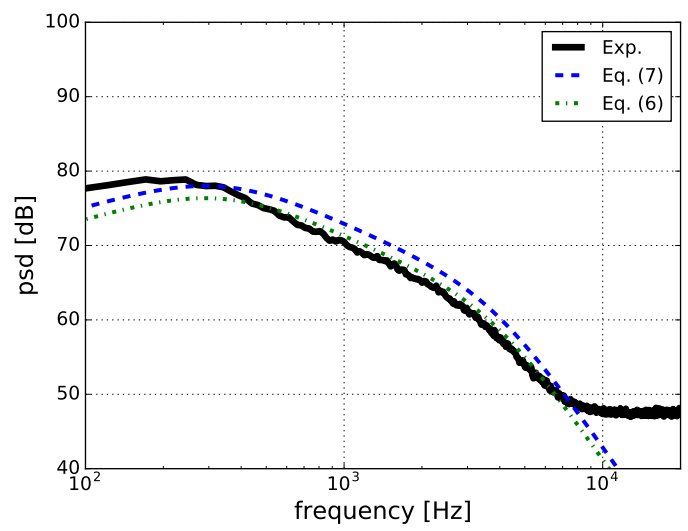

(f)

Figure 9: Predictions of the new model with two expressions for test case of Hu \& Herr; (a) $\mathrm{AOA}=6^{\circ}$, $U_{0}=32.3 \mathrm{~m} / \mathrm{s}$, distance of leading edge $x=1128 \mathrm{~mm}$; (b) AOA $=6^{\circ}, U_{0}=30.8 \mathrm{~m} / \mathrm{s}$, distance of leading edge $x=1210 \mathrm{~mm}$; (c) AOA $=10^{\circ}, U_{0}=32.0 \mathrm{~m} / \mathrm{s}$, distance of leading edge $x=1128 \mathrm{~mm}$; (d) $\mathrm{AOA}=10^{\circ}$, $U_{0}=30.4 \mathrm{~m} / \mathrm{s}$, distance of leading edge $x=1210 \mathrm{~mm}$; (e) AOA $=14^{\circ}, U_{0}=31.2 \mathrm{~m} / \mathrm{s}$, distance of leading edge $x=1128 \mathrm{~mm}$; (f) $\mathrm{AOA}=14^{\circ}, U_{0}=29.9 \mathrm{~m} / \mathrm{s}$, distance of leading edge $x=1210 \mathrm{~mm}$. 


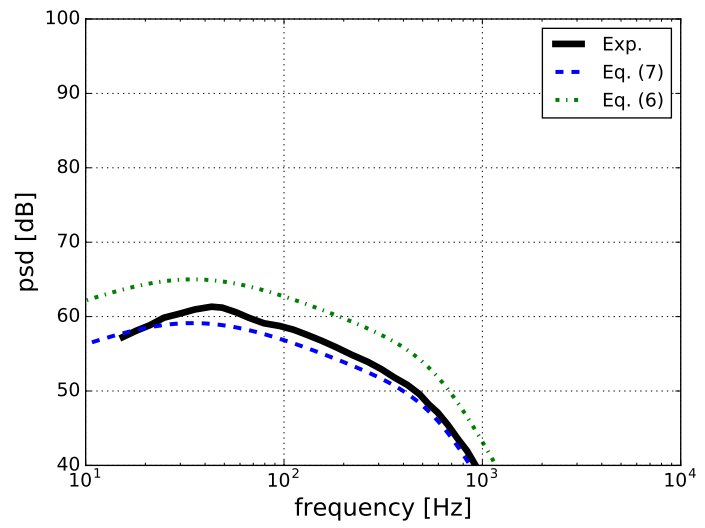

(a)

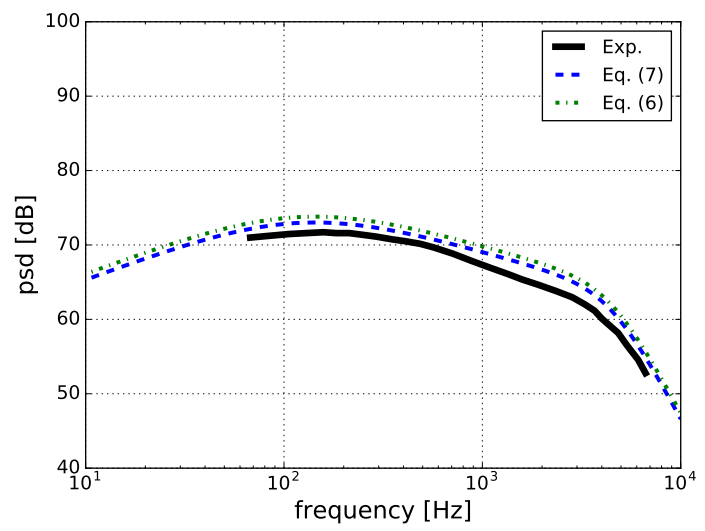

(c)

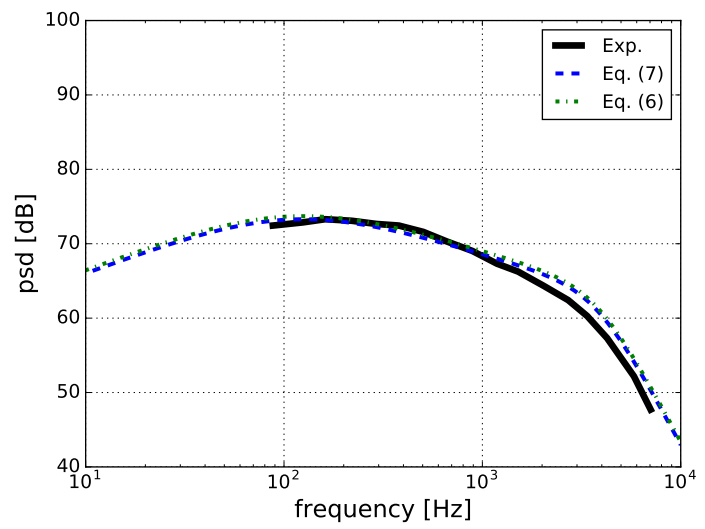

(e)

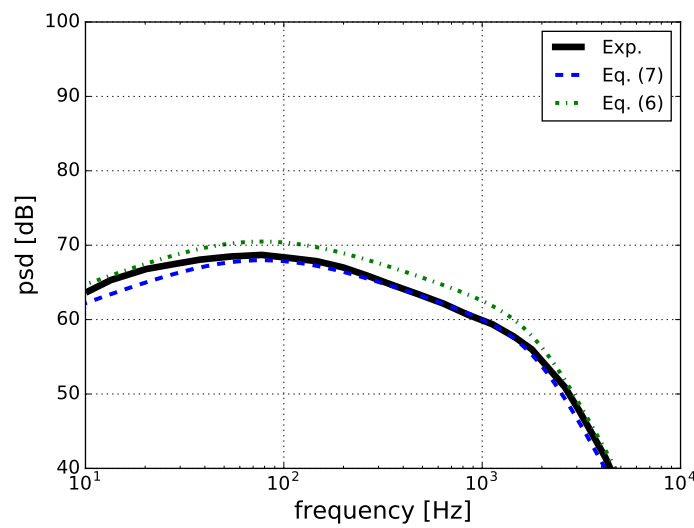

(b)

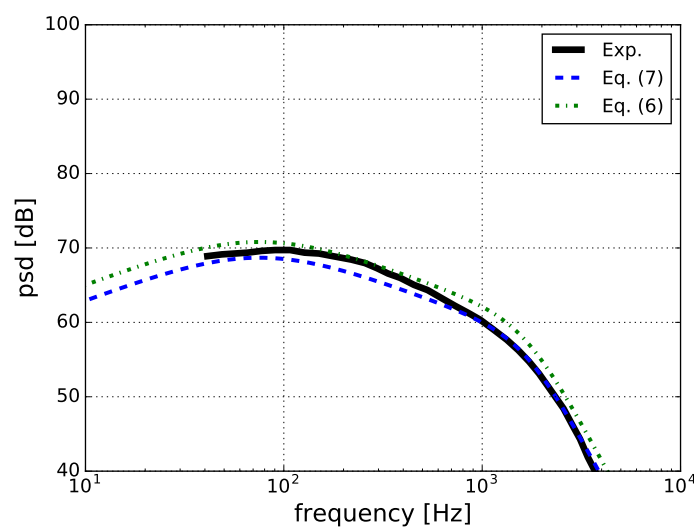

(d)

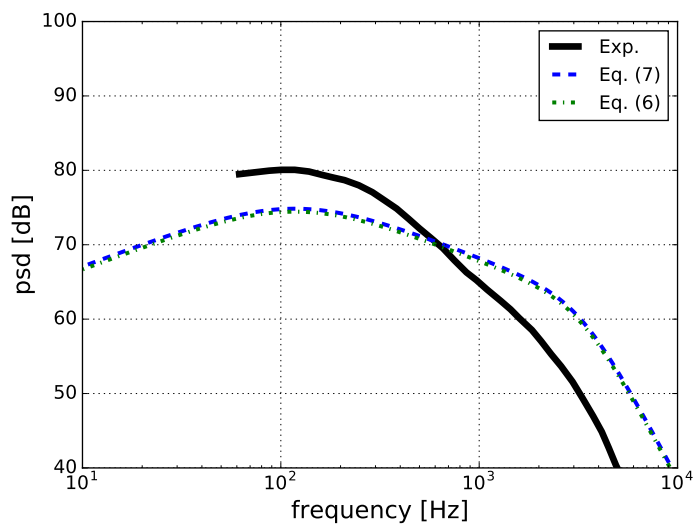

(f)

Figure 10: Predictions of the new model with two expressions for test case of Catlett et al.; (a) open angle $=7^{\circ}, U_{0}=9.0 \mathrm{~m} / \mathrm{s}$, distance of trailing edge $x=50 \mathrm{~mm}$; (b) open angle $=7^{\circ}, U_{0}=18.1 \mathrm{~m} / \mathrm{s}$, distance of trailing edge $x=204 \mathrm{~mm}$; (c) open angle $=7^{\circ}, U_{0}=28.3 \mathrm{~m} / \mathrm{s}$, distance of trailing edge $x=406 \mathrm{~mm}$; (d) open angle $=12^{\circ}, U_{0}=18.4 \mathrm{~m} / \mathrm{s}$, distance of trailing edge $x=154 \mathrm{~mm}$; (e) open angle $=12^{\circ}, U_{0}=28.1 \mathrm{~m} / \mathrm{s}$, distance of trailing edge $x=210 \mathrm{~mm}$; (f) open angle $=17^{\circ}, U_{0}=28.4 \mathrm{~m} / \mathrm{s}$, distance of trailing edge $x=106 \mathrm{~mm}$. 


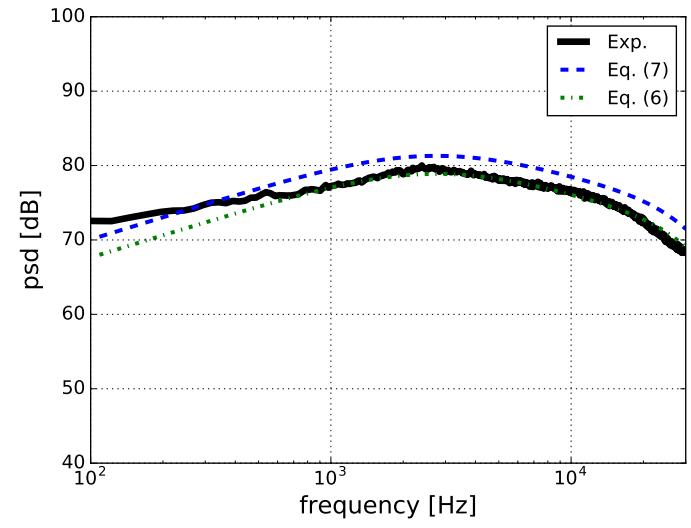

(a)

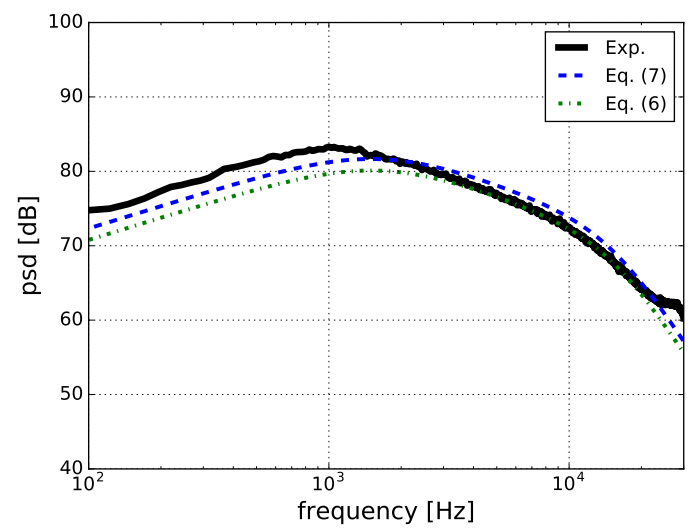

(c)

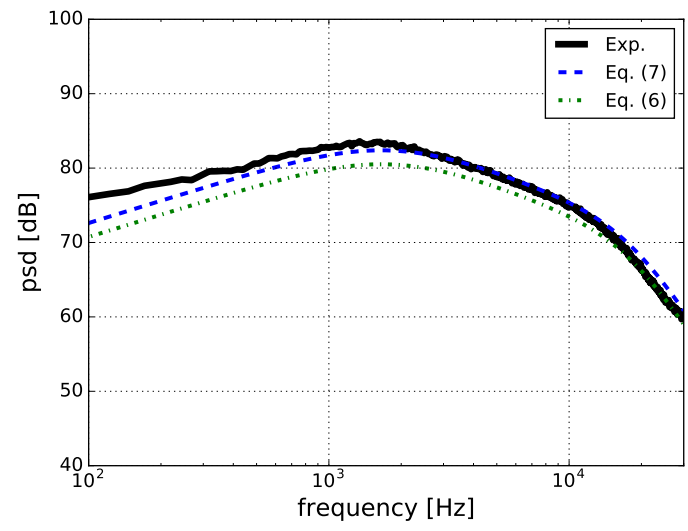

(e)

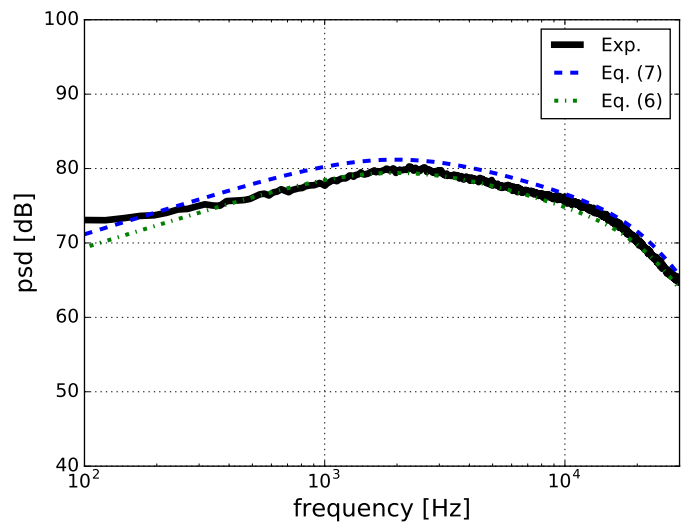

(b)

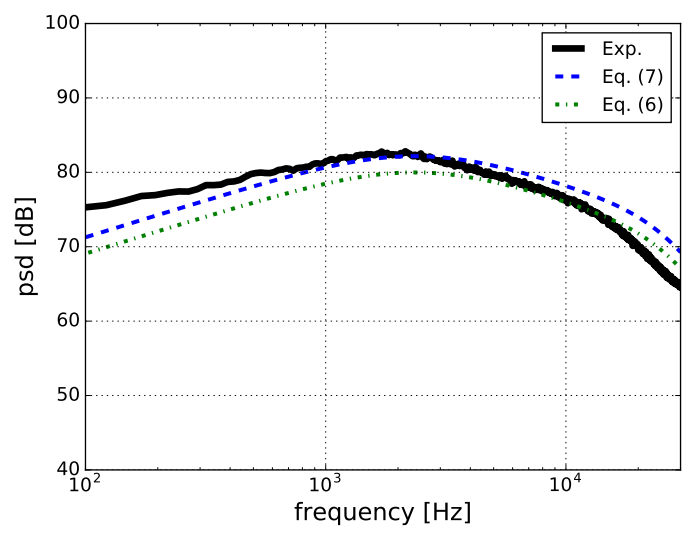

(d)

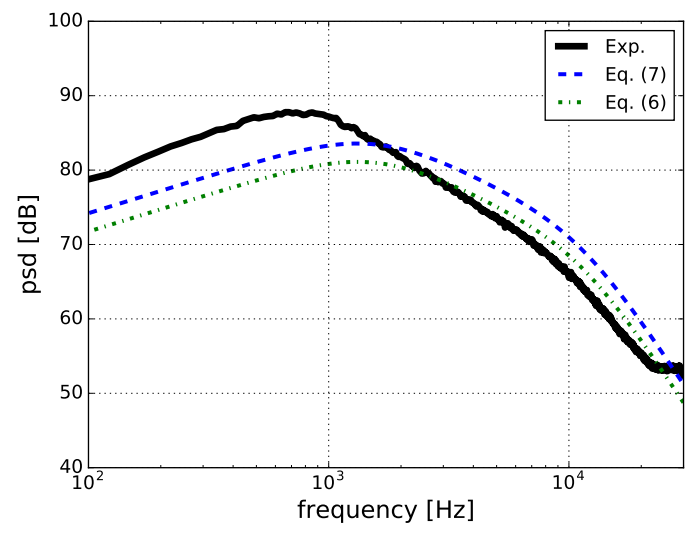

(f)

Figure 11: Predictions of the new model with two expressions for test case of Suryadi \& Herr; (a) $\mathrm{AOA}=-0.8^{\circ}, U_{0}=53.3 \mathrm{~m} / \mathrm{s}$, chord position $x / c=0.77$; (b) $\mathrm{AOA}=-0.8^{\circ}, U_{0}=49.6 \mathrm{~m} / \mathrm{s}$, chord position $x / c=0.88 ;(\mathrm{c}) \mathrm{AOA}=-0.8^{\circ}, U_{0}=45.9 \mathrm{~m} / \mathrm{s}$, chord position $x / c=0.96 ;(\mathrm{d}) \mathrm{AOA}=3.2^{\circ}, U_{0}=54.0 \mathrm{~m} / \mathrm{s}$, chord position $x / c=0.77$; (e) $\mathrm{AOA}=3.2^{\circ}, U_{0}=49.4 \mathrm{~m} / \mathrm{s}$, chord position $x / c=0.88$; (f) $\mathrm{AOA}=3.2^{\circ}$, $U_{0}=45.7 \mathrm{~m} / \mathrm{s}$, chord position $x / c=0.96$. 


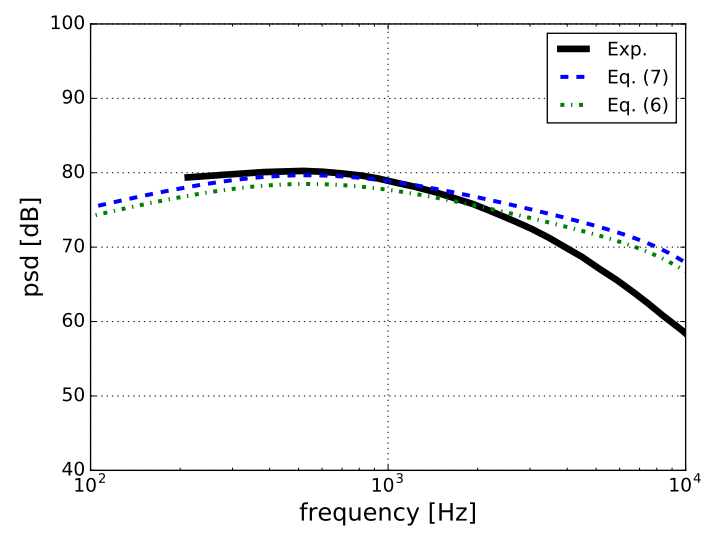

(a)

Figure 12: Predictions of the new model with two expressions for test case of Schloemer, $U_{0}=43.6 \mathrm{~m} / \mathrm{s}$.

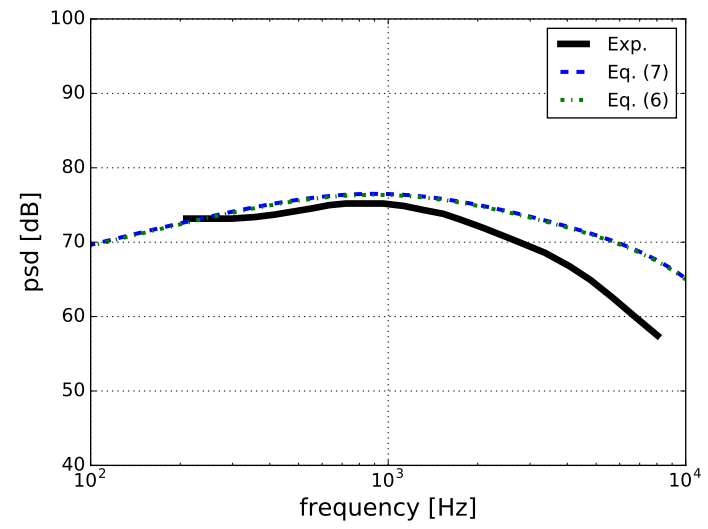

(a)

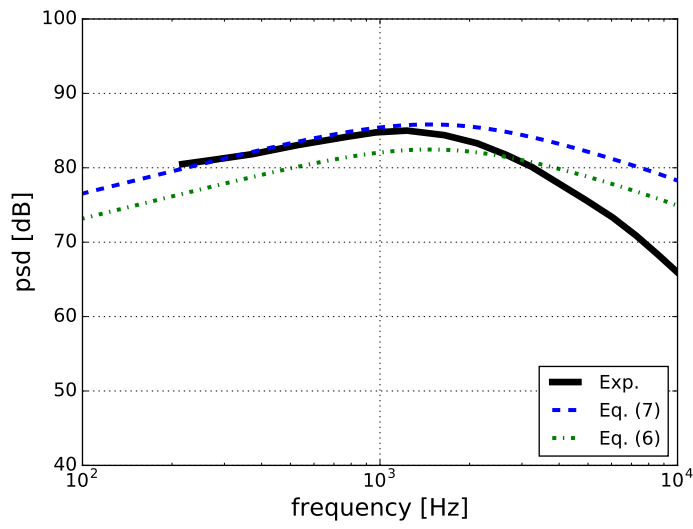

(b)

Figure 13: Predictions of the new model with two expressions for test case of Herrig et al.; (a) $\mathrm{AOA}=0^{\circ}$, $U_{0}=33.4 \mathrm{~m} / \mathrm{s}$, chord position $x / c=0.99$; (b) $\mathrm{AOA}=4^{\circ}, U_{0}=62.7 \mathrm{~m} / \mathrm{s}$, chord position $x / c=0.99$.

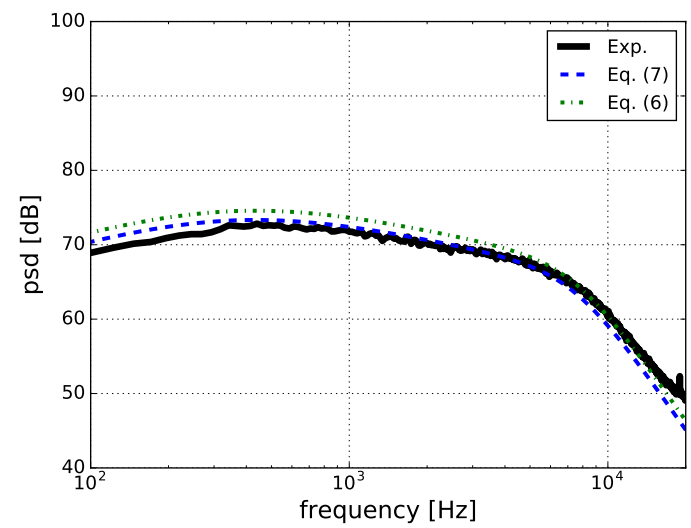

(a)

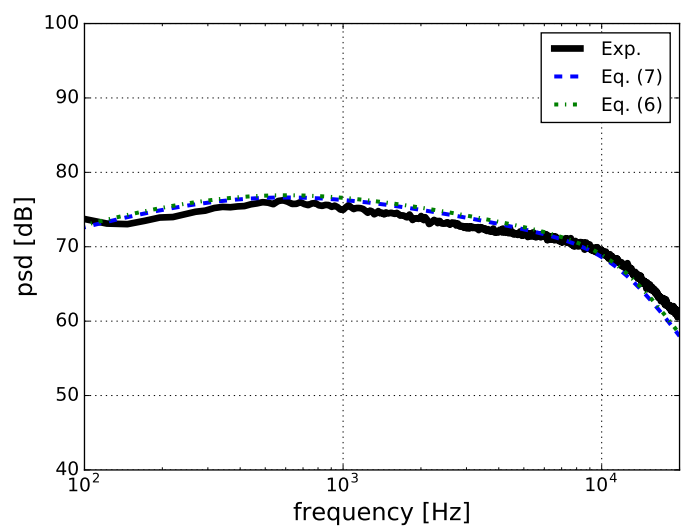

(b)

Figure 14: Predictions of the new model with two expressions for test case of Hu \& Herr for ZPG; (a) $U_{0}=30.2 \mathrm{~m} / \mathrm{s}$, distance of leading edge $x=1210 \mathrm{~mm}$; (b) $U_{0}=39.2 \mathrm{~m} / \mathrm{s}$, distance of leading edge $x=1210 \mathrm{~mm}$. 